\title{
The role of crystallography and the mechanisms associated with migration of incoherent twin grain boundaries
}

\author{
Jonathan L. Priedeman ${ }^{1}$, David L. Olmsted ${ }^{2}$, Eric R. Homer ${ }^{1 *}$ \\ ${ }^{1}$ Department of Mechanical Engineering, Brigham Young University, \\ Provo, UT 84602, USA. \\ ${ }^{2}$ Department of Materials Science and Engineering, University of California, \\ Berkley, CA 94720, USA.
}

\begin{abstract}
With twin grain boundaries playing an important role in numerous materials, it is important to understand their behavior across the full range of possible boundary plane orientations. This work examines the migration of 41 computed $\sum 3$ nickel grain boundaries over a range of temperatures. The boundary plane orientation appears to play the determining role in the nature of the migration observed, which is evident when the data are plotted in the fundamental zone of possible boundary plane

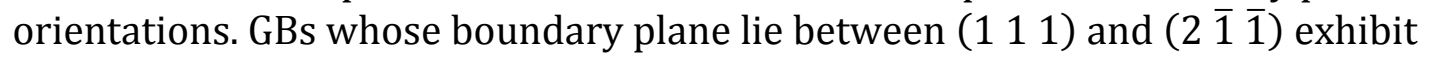
thermally activated migration and the atoms do not move in a coordinated fashion. The remaining GBs, including the (1 0 至) GB, exhibit some form of thermally damped migration. The thermally damped migration is characterized by inverse temperature dependence where the GBs migrate faster at lower temperatures and move in a coordinated fashion involving Shockley partial dislocations. The inverse temperature dependence, which is confirmed by random walk simulations, appears to be consistent with dislocation drag, which could be related to the Shockley partial dislocations. At least one GB exhibits mixed mobility trends due to the presence of both thermally activated and thermally damped migration characteristics.
\end{abstract}

Keywords: grain boundary, twin, grain boundary migration, mobility,

* Corresponding Author: eric.homer@byu.edu 


\section{Introduction}

A recent survey of computed mobility in nickel grain boundaries (GBs) discovered interesting phenomena in incoherent $\Sigma 3$ and other boundaries [1,2]. Select GB types exhibit non-thermally activated mobility, where the mobility is either constant or decreases as temperature is increased. This contrasts with the commonly accepted model of thermally activated grain boundary mobility, where mobility increases as temperature is increased [3]. As an indication that this phenomena is not without precedent, recent work provides examples of different material processes that are non-thermally activated [4]. Nevertheless, the mechanisms that cause this behavior are not resolved, and given that coherent $\Sigma 3$ (twin) GBs play an important role in many different materials, resolving this phenomena requires an understanding of the mechanisms, crystallography, and atomic structures that facilitate these simulated behaviors.

$\Sigma 3$ GBs are of particular importance because they readily manifest in medium to low stacking fault energy materials either through annealing or mechanical twinning [5-7]. The presence of the low energy coherent $\Sigma 3$ interface can lead to enhanced material properties (such as strength [8-10], cracking resistance [6,7,11], and corrosion resistance [12]). Grain boundary engineering (GBE) emerged as a technique to optimize thermomechanical processing routes that enhance properties by increasing the fraction of $\Sigma 3$ and other special GB types [6,7]. While the low energy coherent $\Sigma 3$ boundary interface segments often dominate the microstructure, the incoherent portions of a $\Sigma 3$ GB influence the stability of twins [13,14], indicating a need to understand the full crystallographic dependence of the boundary plane orientation in $\Sigma 3$ GBs.

There are five crystallographic degrees of freedom for a given GB, three for the misorientation between crystals and two for the inclination of the boundary plane between the crystals. While additional atomic degrees of freedom exist (lattice shifts between crystals, boundary placement, atom placement at the boundary, etc.), GBs naturally find a low energy configuration that fixes these microscopic degrees of freedom. In computed GBs, this is accomplished by finding the minimum energy structure among a large number of possible initial configurations [15-17]. In this work, we focus only on the influence of boundary plane orientation, holding the $\Sigma 3$ misorientation constant. This is facilitated by recent publications that detail the fundamental zones (FZs), or unique sets, of boundary plane orientations for a given misorientation $[18,19]$. Thus, one can explore the space of unique boundary plane orientations in a systemic approach.

In the present work, we examine temperature-dependent mobility trends exhibited by $\Sigma 3$ GBs. These various mobility trends (immobile, thermally-activated, and nonthermally activated) show a remarkable dependence on the crystallography as defined by the boundary plane orientation. Representative GBs from each mobility trend are examined in the context of their atomic structure, motion mechanisms, and possible effect these have on the exhibited mobility trends. Finally, origins and implications for each of the mobility trends are discussed. 


\section{Methodology}

The catalog of $\Sigma 3$ nickel GBs investigated here belong to the 388 bicrystal GBs created by Olmsted et al. and compromise all $41 \Sigma 3 \mathrm{GBs}$ that can be created within a periodic box of side length $15 \mathrm{a}_{0} / 2$, with $\mathrm{a}_{0}$ as the lattice parameter [15]. Each of these boundaries is in its minimum energy configuration, determined using the Foiles-Hoyt embedded-atom method (EAM) potential [20]. The method of GB construction and energy minimization is described in detail in reference [15]. The bicrystal simulation cells are periodic in the plane of the GB and non-periodic in the direction normal to the GB. The non-periodic direction has a minimum length of $40 \mathrm{a}_{0} / 2$.

This investigation makes use of the synthetic driving force method for the study of GB migration [21]. In short, the method utilizes an order parameter to distinguish between the atoms in two grains of differing orientation. Additional potential energy is then added to atoms of one grain selected to be the disfavored orientation, while atoms in the other grain have no added energy, making it the favored orientation. The gradient, or difference in energy between the two grains, leads to growth of the grain with the favored orientation and thereby induces grain boundary migration. The applied thermodynamic driving force, $f$, is then obtained by thermodynamic integration [1]. We will refer to the thermodynamic driving force (per unit area) simply as the driving force. The driving force, $f$, and the measured velocity of the GB migration, $v$, are then used to calculate the mobility of the GB, $M$, according to

$$
v=M \cdot f .
$$

The present work augments the previous mobility examination by Olmsted et al., which was limited to temperatures, T, of 600, 800, 1000, 1200 and $1400 \mathrm{~K}$ [1]. Additional GB migration simulations are conducted here over 8 different temperatures ranging from 100 to $600 \mathrm{~K}$. Prior to application of the synthetic driving force, each bicrystal is equilibrated at the test temperature for a minimum of $50 \mathrm{ps}$. The GBs are then driven at different synthetic driving forces, based on maximum potential energy adjustments of $0.005,0.010$, and $0.025 \mathrm{eV} /$ atom. The simulations run until the GB has either migrated more than $50 \AA$ or its immobility sufficiently demonstrated (failure to move in a definite direction for a test of more than $75 \mathrm{ps}$ ). The atomic motions associated with the migration of the different GBs are analyzed primarily using slip vector analysis [22].

The temperature dependence of the GB mobility is then examined to determine whether the GB is immobile or has a thermally activated, or non-thermally activated mobility trend. The thermally activated classification is applied if the mobility follows an Arrhenius law,

$$
M=M_{0} \cdot \exp \left(-Q / k_{B} T\right),
$$

where $Q$ is the activation energy, $k_{B}$ is Boltzmann's constant, and $M_{0}$ is a prefactor with units of mobility. The non-thermally activated mobility trends are classified as athermal, if the mobility is independent of temperature, or thermally damped, if the mobility decreases with increasing temperature with an inverse temperature relationship,

$$
M=M_{0} \cdot 1 / T .
$$


In Equation 3, $M_{0}$ is a prefactor, or proportionality constant, that can vary between different GBs that all have the same temperature dependence. Since the 41 different $\Sigma 3 \mathrm{GBs}$ all have the same misorientation, the crystallographic difference between GBs is limited to the GB plane orientation, described here by the boundary plane, or its normal, in the FZ.

\section{Results}

\subsection{Boundary plane orientation dependence}

While boundary plane orientation has always been recognized as an important determinant of character, the initial classification of the $41 \Sigma 3 \mathrm{GBs}$ into different temperature-dependent mobility trends did not provide insight into the influence of crystallography [19]. However, use of the boundary plane FZ analysis technique $[18,19]$ with the $41 \Sigma 3 \mathrm{GBs}$ indicates a remarkable influence of the crystallography on the temperature-dependent mobility trend; each of the different mobility trends cluster together. The temperature dependent mobility trends are plotted in the boundary plane FZ for the $\Sigma 3$ misorientation in Figure 1. The plot is a stereographic projection of a portion of a sphere, where $\Sigma 3 \mathrm{GBs}$ with boundary plane orientations inside this region have unique character. All boundary plane orientations on the sphere outside this region can be mapped by a symmetry operator of the crystal back into this FZ or irreducible space. It is important to note that the FZ can be defined from the boundary plane orientations from the crystals on either side of the GB plane. Thus, all the vertices of the FZ in Figure 1 are defined for both crystals, marked with a subscript for crystal A or B. However, in all the text, we refer mostly to the boundary plane indices from one crystal (A in Figure 1) without using the subscript, since the second definition is redundant.

The clustering of the mobility trends is as follows. The thermally activated GBs all have boundary plane normals that lie along the lower boundary of the FZ, between the (1 111$)$ and $(2 \overline{1} \overline{1})$ boundary planes. It is noted that the coherent (twin) $\Sigma 3$ is marked as immobile because it never migrated under any of the conditions studied. Nearly all the rest of the GBs in the FZ are non-thermally activated, having thermally damped character. One final GB with normal near the $(2 \overline{1} \overline{1})$, is marked as having mixed mobility trends, for reasons that will be discussed below. This pattern suggests some sort of transition in mobility near the (1 111$)$ and ( $\left.\begin{array}{lll}2 & \overline{1} & \overline{1}\end{array}\right)$ FZ boundary.

This role of the boundary plane was noted in a recent publication, which examined structure-property relationships of various GB types and properties, though the mechanisms leading to the different trends were not discussed [19]. The present work focuses on resolving the mechanisms behind the crystallographic dependence of the mobility trends. To do this, GBs from each mobility trend are examined in detail, with a particular focus on understanding the migration mechanisms and the influence of GB structure. 


\subsection{Thermally Activated GBs}

To understand the observed trends in the GBs that exhibit a thermally activated mobility trend, we first examine the atomic structure of the various GBs and compare their structure to the coherent twin. Each of the thermally activated GBs is identified by the polar angle of its plane normal, relative to the coherent twin. Thus the coherent twin (boundary plane $\left(\begin{array}{lll}1 & 1 & 1\end{array}\right)$ ) has a polar angle of $0^{\circ}$ while the $(2 \overline{1} \overline{1})$ boundary plane has a polar angle of $90^{\circ}$. The various structures are illustrated in Figure 2a, where the view axis is $\left\langle\begin{array}{lll}1 & 1 & 0\end{array}\right\rangle$ and atoms are colored by common neighbor analysis (CNA) and all surrounding FCC atoms have been removed. The twin ( $\left(\begin{array}{lll}1 & 1 & 1\end{array}\right)$ plane) is a twist GB and the $(2 \overline{1} \overline{1})$ plane is a tilt GB, both about the [1 111$]$ axis; all other thermally activated GBs are of mixed character when considered about the [1 111$]$ axis. It can be seen that the lower polar angle GBs have a structure consisting of segments of coherent twin, separated by line defects. This structure is even clearer in boundaries with smaller polar angles than $19.5^{\circ}$. With increasing polar angle the line defects get closer together until they overlap and atomic scale twin segments are no longer observed. However, it is noted that these large polar angle GBs still have individual atoms or rows of atoms that are identified as having an HCP environment according to CNA. The step character of the line defects is clear in Figure 2a. We have not determined the Burger's vector content, if any.

Since these arrays of line defects separated by segments of coherent twin are similar to the Read-Shockley model for GBs created by arrays of dislocations, we fit the GB energies of these thermally activated GBs with the Read-Shockley GB energy equation [23]:

$$
\gamma=E_{0} \theta\left(A_{0}-\ln \theta\right)+E_{C T}
$$

using $E_{0}$ and $A_{0}$ as fitting parameters, and $E_{C T}$ is the energy of the coherent twin. The extra constant, $E_{C T}$, is included, as in other models [24], because the Read-Shockley model is being used as a deviation of the boundary plane, $\theta$, from that of the coherent twin. Typically, the angle $\theta$ refers to a changing misorientation angle. Nonetheless, the regular arrays of defects separating the twin segments, follow the Read-Shockley model. The GB energy values and the Read-Shockley fit $\left(\mathrm{R}^{2}=0.99\right)$ are plotted in Figure $2 \mathrm{~b}$, though it is noted that the (2 $\overline{1} \overline{1}) \mathrm{GB}\left(90^{\circ}\right.$ polar angle) is not included in the fit. The $(2 \overline{1} \overline{1})$ GB shows a very clear deviation from the fit and in examining the structure, one can see that regularly spaced partial dislocations are emerging from one side of the GB. This is consistent with the presence of a region of the 9R structure in GBs near the (2 $\overline{1} \overline{1})$ plane [25-27]. This configuration is a deviation from simple overlapping of line defects that would be predicted by the Read-Shockley model, indicating that the $9 \mathrm{R}$ structural configuration significantly reduces the energy.

Since these GBs follow the Arrhenius law given in Equation 2, their mobility is analyzed as a function of their activation energy. The activation energy is obtained by fitting the measured mobilities for all temperatures at which the GB is mobile; some GBs were not mobile at lower temperatures, indicating a roughening transition [1]. As a result, the coherent twin does not report an activation energy for GB migration because it was never found to be mobile. The activation energies for the remaining 
GBs are plotted in Figure $2 \mathrm{~b}$ as a function of their polar angle, with their $\mathrm{y}$-axis values listed on the right of the graph.

The mechanisms associated with thermally activated GB motion are expected to involve atomic rearrangement; an atom leaving a lattice site from one grain and arriving at a lattice site of the other grain [3]. Figure 3a shows the (2 $\overline{1} \overline{1})$ GB viewed down the $\left[\begin{array}{lll}0 & \overline{1} & 1\end{array}\right]$ axis, demonstrating the smooth transition of the close packed planes across the GB. Figure $3 \mathrm{~b}$ shows the structure of the $\left(\begin{array}{lll}2 & \overline{1} & \overline{1}\end{array}\right) \mathrm{GB}$ viewed down the $\left[\begin{array}{lll}1 & 1 & 1\end{array}\right]$ axis, enabling the stacking sequence across the GB to be examined. Figure $3 \mathrm{c}$ contains magnified views of the atom positions of the two grains, drawing attention to the atom position reversal across the GB: the $\mathrm{ABC}$ order of the top grain becomes $\mathrm{ACB}$ in the bottom grain, which is consistent with a $\Sigma 3$ misorientation. In these three images, the color scheme of the atom markers relates to the stacking plane to which they belong (A, B and C corresponding, respectively, to black, purple and yellow). The atom marker shape conveys the categorization of that atom by CNA, with atoms in a local FCC structure represented as circles, atoms in a local HCP structure represented as triangles, and atoms with no identifiable local structure as squares.

To examine the nature of the atomic motions that enable migration, the slip vector [22] is utilized to examine the migration of the (2 $\overline{1} \overline{1})$ GB under a driving force of 0.01 $\mathrm{eV} /$ atom at $1200 \mathrm{~K}$. Results from the slip vector analysis are illustrated in Figure 3d for a portion of the GB, where the slip direction of each atom is plotted with an arrow. Once again, the view axis is the [1 111 ] direction, and the slip vectors are colored according to their corresponding stacking plane, consistent with the rest of the figure.

To accomplish GB migration in the [2 $\overline{1} \overline{1}]$ into the top grain, the stacking sequence of the top grain must be reversed to match that of the bottom grain. But since there is no overall translation of the atoms, the net shift of any local region must be zero. To better discern patterns in the directionality of the slip vectors, the slip vectors are plotted as angular distributions according to specific planes in Figure 3e. In this plot, distance from the origin is indicative of the fraction of shifting atoms moving in that direction. The plot also indicates the $\langle 211\rangle$ directions that correspond to the direction of the Burger's vector of a Shockley partial dislocation. The three stacking planes move relative to one another. Atoms belonging to the "B" plane shift laterally relative to the GB migration. The atoms of the " $\mathrm{A}$ " plane tend to shift with the direction of GB migration, while the atoms of " $\mathrm{C}$ " move opposite the direction of migration. It is interesting to note that the "B" plane with no HCP atoms at the GB (c.f. Figure 3a) moves laterally, while the other stacking planes with HCP atoms move with or oppose the general direction of GB migration; however, this is noted as a possible correlation and is not meant to suggest causation.

As indicated by Figure 3a, each of the planes have specific roles in the migration in order to reverse the stacking sequence as it migrates. The net direction must be zero because the atoms are not migrating long distances. More importantly, the directions of the slip vector are contained within a single plane and are fairly diffuse over a wide range of angles. Thus, the planar motions relative to each other are coordinated, but the individual motions in the plane have no simple description. This is generally consistent with the thermally activated motion observed by O'Brien and Foiles for two 
boundaries, one $\Sigma 5$ and one $\Sigma 15$, where they observed a complex shuffle of the atoms as the mechanism [28].

\subsection{Non-Thermally Activated: Thermally Damped GBs}

The non-thermally activated mobility trend dominates the $\Sigma 3$ GBs as 30 of the 41 GBs exhibit the thermally damped trend. In the original examination of mobility trends temperatures were limited to 600-1400 K, though all 30 thermally damped GBs were still mobile at $600 \mathrm{~K}$. In the present work, the same GBs are tested for mobility down to $100 \mathrm{~K}$, where they are all still mobile. To illustrate the mobility over these temperature ranges, we plot the data in two ways for three GBs that are representative of the 27 other thermally damped GBs. In the top row of Figure 4, the data are plotted as the natural log of mobility as a function of the natural log of temperature. The bottom row plots velocity as a function of driving force divided by temperature. These two plots illustrate different characteristics of the thermally damped motion.

The top plots of the natural log of mobility in Figure 4 emphasize the thermally damped trend given by Equation $3, \ln M=\ln M_{0}-1 \cdot \ln T$, where the -1 slope is the inverse exponent in Equation 3 and $\ln M_{0}$ is the y-intercept in this plot. At high $\mathrm{T}$, all three GBs exhibit the inverse temperature dependence, and at low T, the mobility for all three becomes athermal, or independent of temperature. Note that in all three cases, the athermal value of the mobility is dependent upon the magnitude of the driving force. The (13 $4 \overline{5}$ ) and (11 85 ) GBs are included to show the extreme examples of high and low of $M_{0}$, respectively. The ( $\left.\begin{array}{lll}3 & 0 & \overline{1}\end{array}\right)$ is included to illustrate that 5 of the 30 thermally damped GBs exhibit anomalous behavior in the behavior change from thermally damped at high $\mathrm{T}$ to athermal at low $\mathrm{T}$. It is noted that these $5 \mathrm{GBs}$ are all near the $(7 \overline{3} \overline{4}) \mathrm{GB}$, which is classified as having mixed mobility and will be discussed in the following section.

As noted, the mobility in the top plots appears to have a significant driving force dependence at low T, where it becomes athermal. For reasons that will become apparent, we choose to plot the same data as velocity, $v$, as a function of the ratio of driving force over temperature, $f / T$. This plot style is adopted from an examination of the influence of driving force and temperature on the velocity of dislocations [29]. These bottom plots of velocity in Figure 4 emphasize the combination of Eqs. 1 and 3, which suggests $\mathrm{v} \propto f / \mathrm{T}$. As can be seen in Figure 4, this relationship holds true for low values of $f / T$, which is essentially dominated by the high temperatures, $T$. But this relationship breaks down when the velocity saturates and becomes independent of $f / T$ at low $\mathrm{T}$.

Note that in the velocity plots, the different mobility saturation values nearly merge to a single velocity saturation value. The inverse temperature dependence is inherent in these plots because the x-axis is $f / T$ and $M_{0}$ is the slope of the data in the linear regime at low $f / T$.

To compare the salient features of the data, we examine the values of proportionality constant, $M_{0}$, the magnitude of the saturation velocity, and the magnitude of $\mathrm{f} / \mathrm{T}$, when the mobility behavior changes from thermally damped at high $\mathrm{T}$ to athermal at low $\mathrm{T}$. 
These features of the velocity plot are identified in the illustration in Figure 5a. Figures $5 b-5 d$ plot the behavior change point, identified as a specific $f / T$ ratio, from low temperature athermal behavior to high temperature thermally damped behavior, the magnitude of the saturation, or athermal, velocity, and the thermally damped proportionality constant $M_{0}$, respectively, all of which are plotted in the FZ. To simplify the presentation, the data for Figure 5 are taken for the $0.01 \mathrm{eV} /$ atom driving force simulations.

Figures $5 b-5 d$ yield several important results. The proportionality constant, $M_{0}$, and the velocity saturation are relatively smooth across the FZ, suggesting that these factors depend on the crystallography of the GB. The velocity saturation reaches a maximum at a boundary plane normal of [1 $\overline{3} 1]$, though the exact reason for this is unclear. In examining Figure 5b, it is less clear that a relation exists between crystallography and the value of $f / T$ where the saturation occurs. We should point out, however, that something more complex may be going on for a handful of boundary plane normals near the $(7 \overline{4} \overline{3}$ ), which exhibits mixed mobility trends. These GBs overshot the saturation velocity before decreasing down to saturation, as shown in Figure 4 for the ( $\left.\begin{array}{lll}3 & 0 & \overline{1}\end{array}\right)$ GB.

To better understand the mechanisms associated with the thermally damped GB migration, we first examine the structure of a typical thermally damped GB. The atomic structure for the (1 0 兵) GB is illustrated in Figure $6 \mathrm{a}$ and $6 \mathrm{~b}$, with views from the $\left[\begin{array}{lll}1 & \overline{2} & 1\end{array}\right]$ and the $\left[\begin{array}{lll}1 & 1 & 1\end{array}\right]$ axes, respectively. The atoms are colored by their stacking plane and have symbols that indicate their CNA classification, as in Figure 3. Figure 6a demonstrates the smooth transition of atomic planes across the GB and Figure $6 \mathrm{~b}$ enables examination of the stacking sequence across the GB. Magnified views of the stacking sequences of the two grains are found in Figure $6 \mathrm{c}$ to highlight the reversal of two of the stacking planes.

The atomic motions associated with the migration are again illustrated using the slip vector. Figure $6 \mathrm{~d}$ presents the slip vectors for the $\left(\begin{array}{lll}1 & 0 & \overline{1}\end{array}\right) \mathrm{GB}$ under a driving force of $0.01 \mathrm{eV} /$ atom at $200 \mathrm{~K}$. With a [ 1111 ] view axis the slip vectors match the color

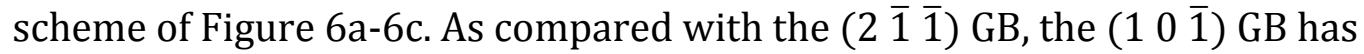
considerably more ordered slip vectors.

Similar to Figure 3e, Figure 6e presents an angular distribution plot of the slip vectors for specific planes. It can be seen that the atoms of the three stacking planes segregate their motion to distinct and specific directions, or nearby ranges, concentrated around the $\langle 211\rangle$ directions. Whereas the thermally activated atom motion during GB migration had each plane doing something different, the thermally damped atom motions have each plane moving in very similar patterns but in different directions, since the distributions for each stacking plane are roughly identical. This behavior is similar to the anti-thermal motion of a $\Sigma 7$ GB observed by O'Brien and Foiles, in that it also involved in-plane coordinated motions, though the mechanism differs significantly from that observed here [28].

Migration of incoherent $\Sigma 3$ GBs has long been known to involve the migration of partial dislocations [14,30-33], so the results of the slip vector analysis are not 
surprising. However, recent work has also indicated that this motion can occur at fast speeds $[34,35]$ and may be responsible for detwinning at cryogenic temperatures $[36,37]$, both of which are consistent with the characteristics of the thermally damped motion detailed here. The dislocation mechanism may also be the origin for the temperature-dependence of the mobility, which is considered in the discussion section.

\subsection{Mixed Mobility Trend}

The original temperature-dependent mobility study classified one GB, boundary plane $(7 \overline{3} \overline{4})$, as athermal because the variation in mobility over the temperature range 600$1400 \mathrm{~K}$ was minimal. In the present work, this GB is identified as having mixed mobility for reasons that are explained here. The mobility over this larger temperature range is presented in an Arrhenius plot in Figure 7a, and the boundary is found to be mobile down to $100 \mathrm{~K}$. Because of the scaling in Figure 7a, the data points at the highest 5 temperatures are hard to discern. However, the trend that once appeared athermal now appears to be thermally activated. Using the Arrhenius Equation 2, the activation energy is found to be $0.009 \mathrm{eV}$. With such a small activation energy, it is not surprising that the original study over a smaller temperature range classified the GB as having athermal mobility. However, in examining Figure 7a further, it is important to note that the mobility data does have one feature not characteristic of thermally activated migration. At low temperatures, the lower driving forces lead to higher mobility values, which is a characteristic of the GBs that exhibit thermally damped mobility, as in Figure 4. Thus, this GB has characteristics that are consistent with both thermally activated and thermally damped GBs. This mixed character, or lack of conformance to a single motion mechanism, is highlighted further in Figure 7b, which plots the velocity of the GBs as a function of the driving force over temperature, $f / T$. In contrast to the plots in Figure 4 for the $(134 \overline{5})$ and (11 8 5) GBs, there is no indication of a saturation velocity independent of driving force. But in common with the (3 $0 \overline{1})$ in Figure 4, there is a spike, or quick rise and fall in the velocity as $f / T$ is increased. The full behavior of this GB is not clear, though if there is any sort of transition from one mechanism to another, this spike may be indicative of the transition between the two.

To examine the mechanisms of migration for this GB, the atomic structure and slip vector are again analyzed and presented in Figure 8 . Figures $8 \mathrm{a}$ and $8 \mathrm{~b}$ contain views of the atomic structure down the [ $\left.\begin{array}{lll}1 & \overline{11} & 10\end{array}\right]$ and [ $\left[\begin{array}{lll}1 & 1 & 1\end{array}\right]$, respectively. The representation scheme throughout this figure is consistent with the scheme employed in Figures 3 and 6. The atomic planes vary smoothly across the GB as seen in Figure $8 \mathrm{a}$, and Figure $8 \mathrm{~b}$ shows that the structure of the GB is comprised of short segments of the $(2 \overline{1} \overline{1})$ GB structure along with line defects separating these facets. The magnified views of the atomic structure for both grains in Figure 8(c) again indicate the reversal of planes across the GB. The slip vectors of the $(7 \overline{3} \overline{4})$ GB are shown in Figure 8(d) for migration under a driving force of $0.010 \mathrm{eV} /$ atom at $200 \mathrm{~K}$. The directionality of the slip vectors indicates an ordered slip mechanism like that of the thermally damped GB discussed in Figure 6. The angular distributions of the slip vectors for atoms that did participate are plotted in Figure 8(e). The similarity of the distributions (in shape and 
adherence to the 2211$\rangle$ directions) to the distributions of the thermally damped boundary of Figure 6 provides another indication that there is strong thermally damped behavior influencing the migration mechanisms of this particular GB.

This GB supports the conclusions by O'Brien and Foiles that one cannot determine a mechanism simply by the temperature-dependence of the GB mobility [28]. At the temperature where the ordered slip vectors were observed, the mobility is slightly thermally activated, with an activation energy of $0.009 \mathrm{eV}$. One or more mechanisms may be present and any one of these may exhibit a dominant role depending on temperature or other conditions [28,38].

\section{Discussion}

\subsection{The role of crystallography on GB migration}

The mobility of the different $\Sigma 3$ GBs shows very clear dependence upon the boundary plane orientation. This dependence is strong enough to determine whether a GB is thermally activated or thermally damped, or whether the thermally damped GB will have relatively high or low mobility as compared with other GBs of the same classification.

In the thermally damped GBs, the mobility proportionality constant and the velocity saturation value are both smooth functions in the FZ, as shown in Figure 5. The

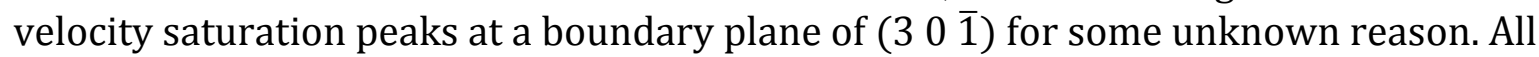
the GBs show the same slip vector analyses indicating ordered motion.

The thermally activated GB nearest (lllll) consists of segments of the $\left(\begin{array}{lll}1 & 1 & 1\end{array}\right)$ boundary decorated with line defects. Since the $\left(\begin{array}{lll}1 & 1 & 1\end{array}\right)$ boundary is immobile, it seems plausible that the motion of this boundary is controlled by motion of the line defects along the grain boundary. The activation energy slowly rises with increased density of these line defects. However, there is a discontinuity in the activation energy for the $(2 \overline{1} \overline{1})$ GB. The more complex, but lower energy structure of this GB requires more energy to move.

The mixed-mobility ( $7 \overline{3} \overline{4}$ ) GB exhibits characteristics of both thermally activated and thermally damped mobility because it is comprised of mostly ( $2 \overline{1} \overline{1}$ ) atomic structures with line defects. Furthermore, the 5 GBs that are near the $(7 \overline{3} \overline{4})$ boundary plane and exhibit a non-smooth transition from high temperature thermally damped motion to low temperature athermal motion also have large regions of (2 $\overline{1} \overline{1})$-like atomic structure.

The crystallography sufficiently constrains the atomic structure of the GBs such that GBs that have certain features behave in a similar fashion. Recent work has studied larger $\Sigma 3$ GBs and found them to facet into one of the three low energy boundary planes, ( $\left.\begin{array}{lll}1 & 1 & 1\end{array}\right),\left(\begin{array}{lll}2 & \overline{1} & \overline{1}\end{array}\right)$, and ( $\left.\begin{array}{lll}1 & 0 & \overline{1}\end{array}\right)$, that lie at the vertices of the FZ [39], which faceting is predicted [40]. In this work, any time the (1 0 1) thermally damped facet is present, its faster motion dominates the migration of the GB. In this work, the GBs are too small to have facets, but exhibit similar behaviors. Any GB with a line defect or step 
that allows the Shockley partials to move in an ordered manner indicated in Figure 6 dominates the migration and the GB moves fast as a result. The crystallographic difference between the thermally activated and thermally damped GBs must then be the presence of line defects or steps that enable the Shockley partials to cause the GB to migrate.

In one curious comparison with experiments, while the simulations indicate that the (1 $\left.0 \begin{array}{ll}1 & 1\end{array}\right)$ facets provide the faster motion, $\left\{\begin{array}{lll}2 & 1 & 1\end{array}\right\}$ facets are observed to migrate in at least two cases $[14,41]$. Further work is required to understand this apparent anomaly.

\subsection{Thermally damped migration mechanism}

While the crystallography explains the variation of the mobility across the FZ, the thermally damped mobility is still a curious phenomenon that is not expected in GB migration. As noted in the introduction, recent work does point out various material processes that do not conform to Arrhenius, or thermally activated, temperature dependence [42]. Athermal GB migration has also been reported in experiments $[3,43,44]$. Nevertheless, the thermally damped migration mechanism deserves further attention.

Of prime importance is whether the method by which the boundary migrates influences the mechanisms or temperature-dependence of the boundary. GB migration has been proven to be independent of the nature of the driving force in several cases $[45,46]$. We further confirm this independence by employing random

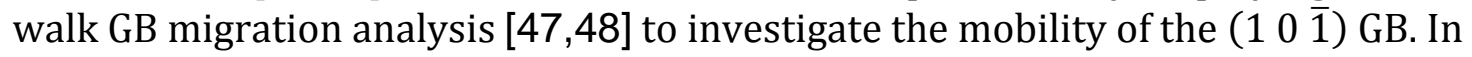
random walk analysis, one can estimate the mobility, $M$, of a given GB by observing the fluctuations of the boundary position, $\bar{x}$, over different time intervals, $t$, and temperatures, $T$. Based on these fluctuations, the mobility is defined as

$$
M=\frac{A}{2 k T} \cdot \frac{d\left\langle\bar{x}^{2}\right\rangle}{d t}
$$

where $A$ is the interface area, $k$ is Boltzmann's constant, and $\left\langle\bar{x}^{2}\right\rangle$ is the mean squared displacement of the $\mathrm{GB}$, of which the mobility is a time derivative of this quantity. We obtain $\left\langle\bar{x}^{2}\right\rangle$ by examining 200 unique simulations of the same GB, but similar to Deng and Schuh, we also get multiple measurements of the GB displacement from each simulation by using different times to obtain the reference position for the displacement of the GB [48]. The reference positions of the GB in a single simulation are separated by $500 \mathrm{fs}$. The displacement of the GB is measured over periods of 0.5 ps to 10 ps in steps of $0.5 \mathrm{ps}$. $\left\langle\bar{x}^{2}\right\rangle$ is then calculated by combining the boundary displacements from the 200 measurements of each of the 200 simulations at different time steps. The mobility, $M$, is then obtained by fitting the slope of $\left\langle\bar{x}^{2}\right\rangle$ as a function of time , as indicated in Figure 9 a for the $200 \mathrm{~K}$ simulation. In all cases, $M$ was fit over the period of 3-10 ps to capture the linear (diffusive) regime, leaving out the early transient response in the first 3 ps. Since each point of $\left\langle\bar{x}^{2}\right\rangle$ represents a distribution of the boundary position fluctuations, we plot three of these distributions as cumulative distributions, with the overlaid normal cumulative distribution function. The good agreement between the distribution and function give us confidence in each 
of the $\left\langle\bar{x}^{2}\right\rangle$ measurements, and the linear trend in $\left\langle\bar{x}^{2}\right\rangle$ give us confidence in the calculated mobility values.

Figure $9 \mathrm{~b}$ provides a comparison of the random walk mobility measurements with the mobility measurements obtained from the synthetic driving force. While the mobilities reported by the two methods do not match perfectly, the thermally damped trend, or -1 slope, is clearly present in both. Interestingly, the random walk analysis, which has no driving force, shows no indication of saturation, which matches the driving force dependence of the synthetic driving force data, where the lower the driving force, the lower the temperature at which saturation occurs. The difference in mobility values between the random walk and synthetic driving force method may be attributed to the fact that recent work has developed an improved synthetic driving force method, called the energy conserving orientational (ECO) driving force [49]. The ECO driving force method solves minor flaws in the synthetic driving force method, but it is noted that while actual magnitudes of mobility change between the new ECO driving force and the old synthetic driving force methods, the temperaturedependence of the mobility trends does not change [49]. As such, the most important feature to note in the random walk analysis is that the inverse temperature dependence of the mobility is clear. The thermally damped migration mechanisms is a real observation of GB migration simulated by molecular dynamics.

One possible origin for the inverse temperature dependence of the thermally damped GB migration may result from the ordered slip mechanism illustrated in Figure 6e. In examining Figure 6e further, it is apparent that the slip vectors are dominant in the $\langle 112\rangle$ directions. This motion is consistent with the description of triplets of Shockley partials on the three stacking planes, whose motion has been observed in simulation and experiments of migrating incoherent $\Sigma 3$ GBs [14,39,41,50]. The remaining discussion centers around dislocation mediated migration since this motion is facilitated by Shockley partial dislocations.

Dislocation interact with phonons and various other lattice phenomena, which can lead to drag or slowing of dislocation motion. Many of these phenomena have very distinct temperature dependencies [51-55]. In our classical simulations, both electronic and quantum effects are absent. Above about half the Debye temperature, phonon drag, which is present in our simulations, predominates. Phonon drag is proportional to the phonon density, and so proportional to the temperature. Since the velocity of a dislocation is inversely proportional to the drag coefficient $(v \propto 1 / B)$ and the mobility of a GB is directly proportional to the velocity $(M \propto v)$, the mobility becomes inversely proportional to the drag coefficient $(M \propto 1 / B)$. Thus, at temperatures above half the Debye temperature, half of $450 \mathrm{~K}$ for $\mathrm{Ni}$ [56], GB migration facilitated by dislocation motion could be expected to exhibit an inverse temperature dependence of its mobility $(M \propto 1 / T)$ if dislocation drag mechanisms are active.

In classical simulations, like ours, the inverse temperature dependence would be expected at low temperatures, but instead, the mobilities reach a maximum value and become athermal. However, the random walk simulations show that in the absence of a driving force, this inverse temperature dependence does continue as the 
temperature drops to $100 \mathrm{~K}$. Furthermore, the synthetic driving force results show a clear driving force dependence at low temperatures, with higher mobility at lower

driving force. This does not explain why the velocities saturate at values of $4-20 \%$ of the lowest wave speed. In, for example, work by Olmsted et al, dislocation velocities saturate at magnitudes near the lowest wave speed [29,57]. If the mechanism for the motion of the boundary is through motion of Shockley partial dislocations in the grain boundary, and the motion of the Shockley partials is in $\{111\}$ planes, as expected, it is possible to compute the velocity of the Shockley partials from the velocity of the boundary and the angle between the $\{111\}$ planes and the boundary normal. It this case the motion of the assumed Shockley partials saturates at velocities up to $\sim 70 \%$ of the lowest wave speed. If in all cases the implied saturation velocity for the assumed Shockley partials was this high, the velocity saturation of the GBs would be explained. Unfortunately, the ratio is not consistently this high.

However, the velocity saturation may not be important to actual behavior at typical driving forces. As shown in Figure 9b, the velocity of the boundaries in the synthetic driving force simulations reach the saturation velocity at lower temperatures as the driving force is lowered. If the driving force were lowered further, the data would trend towards all being in the linear portion of the curve, and it might never saturate. This would be consistent with the zero driving force limit of the random walk simulations, which have an inverse relationship of mobility and temperature down to $100 \mathrm{~K}$. Furthermore, since driving forces of simulations are typically higher than that of experiments $[3,21,48,58]$, the low driving force limit is likely more representative of what will be observed in real life, where the velocity saturation is not likely to be reached.

In short, it is plausible that the thermally damped nature of $\Sigma 3$ GB migration is caused by the dislocation mediated migration of the GB by triplets of Shockley partial dislocations. This would, in turn, cause the $\Sigma 3$ GB mobility to have the same inverse temperature dependence as dislocation drag.

\subsection{System size dependence}

It is important to recognize that these simulations have small cell sizes. Recent work has demonstrated that cell size plays an important role in the migration characteristics of simulated boundaries [59-61]. Small sizes can complicate nucleation of migration steps that only exist on large length scales. More recent analysis of $\Sigma 3 \mathrm{~GB}$ migration simulations have shown that cell size does indeed play a role in affecting the mobility of the GBs, though the temperature-dependence of the trends, and the Shockley partial dislocation mechanism, remain largely unaffected [50]. Furthermore, larger simulation cells show faceting consistent with experiments and mobility trends consistent with the present work [39]. Additionally, imperfections inserted into a $\Sigma 3$ GB only act to accelerate the migration rate, rather than slow it down [50].

\section{Conclusions}

Twin boundaries play an important role in the behavior of numerous materials and are influence by both the coherent and incoherent facets of the $\Sigma 3$ boundary. This 
work examines the role of crystallography as it affects the migration mechanisms of different $\Sigma 3$ GBs.

Boundary plane orientation appears to play a definitive role in determining the variation of grain boundary mobility of $\Sigma 3 \mathrm{GBs}$. The coherent twin is found to be immobile in all cases. GBs with boundary plane orientations between (111) and

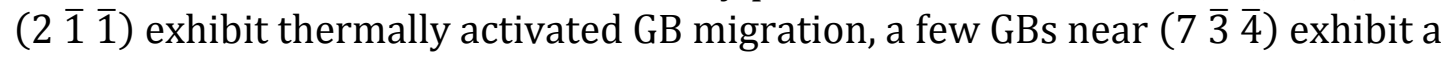
combination of thermally activated and thermally damped GB migration, and the remainder of the GBs, which account for most of the possible boundary plane orientations, exhibit thermally damped mobility. The crystallographic dependence of these trends is particularly evident when they are examined in the fundamental zone of boundary plane orientation.

As expected, the thermally activated mobility is characterized by an Arrhenius dependence on temperature. The atomic motion of this GB migration is characterized by a complex rearrangement of the atoms at the boundary that lacks the clear pattern of the motion of the thermally damped GBs.

Thermally damped mobility is characterized by an inverse temperature dependence on mobility, where mobility decreases as temperature is increased. An examination of the atomic motion mechanisms indicates that this behavior is facilitated by triplets of Shockley partial dislocations. Random walk simulations confirm the thermally damped mobility temperature dependence.

\section{Acknowledgements}

This work is supported by the U.S. Department of Energy, Office of Science, Basic Energy Sciences under Award \#DE-SC0016441. 


\section{References}

[1] D.L. Olmsted, E.A. Holm, S.M. Foiles, Survey of computed grain boundary properties in face-centered cubic metals-II: Grain boundary mobility, Acta Materialia. 57 (2009) 3704-3713. doi:10.1016/j.actamat.2009.04.015.

[2] E.R. Homer, E.A. Holm, S.M. Foiles, D.L. Olmsted, Trends in grain boundary mobility: Survey of motion mechanisms, Jom. 66 (2014) 114-120. doi:10.1007/s11837-013-0801-2.

[3] G. Gottstein, L.S. Shvindlerman, Grain Boundary Migration in Metals, CRC Press, Boca Raton, 2010.

[4] E.A. Holm, Unraveling the causes of fast grain boundary motion, in: Understanding Grain Boundary Migration 2015 Conference, Günzburg, Germany, 2015 .

[5] M.V. Klassen-Neklyudova, Mechanical Twinning of Crystals, Springer, New York, 1964 .

[6] V. Randle, Grain boundary engineering: an overview after 25 years, Mater Sci Tech-Lond. 26 (2010) 253-261. doi:10.1179/026708309X12601952777747.

[7] T. Watanabe, S. Tsurekawa, X. Zhao, L. Zuo, The Coming of Grain Boundary Engineering in the 21st Century, in: A. Haldar, S. Suwas, D. Bhattacharjee (Eds.), Microstructure and Texture in Steels, Springer London, London, 2009: pp. 43-82. doi:10.1007/978-1-84882-454-6_4.

[8] Y. Zhang, J. Wang, H. Shan, K. Zhao, Strengthening high-stacking-fault-energy metals via parallelogram nanotwins, Scripta Mater. 108 (2015) 35-39.

doi:10.1016/j.scriptamat.2015.05.039.

[9] Y. Karasawa, T.-F.M. Chang, A. Shibata, M. Sone, Strengthening effect of twin boundaries in bcc crystal evaluated through a micro-bending test, MRS Proc. 1297 (2011) mrsf10-1297-p10-32. doi:10.1557/opl.2011.6oo.

[10] P. Gu, M. Dao, Y. Zhu, Strengthening at nanoscaled coherent twin boundary in f.c.c. metals, Philos Mag. 94 (2014) 1249-1262. doi:10.1080/14786435.2014.885138.

[11] B.W. Reed, M. Kumar, R.W. Minich, R.E. Rudd, Fracture roughness scaling and its correlation with grain boundary network structure, Acta Materialia. 56 (2008) 3278-3289.

[12] M. Shimada, H. Kokawa, Z.J. Wang, Y.S. Sato, I. Karibe, Optimization of grain boundary character distribution for intergranular corrosion resistant 304 stainless steel by twin-induced grain boundary engineering, Acta Materialia. 50 (2002) 2331-2341. doi:10.1016/S1359-6454(02)00064-2.

[13] R.L. Fullman, J.C. Fisher, Formation of Annealing Twins During Grain Growth, J Appl Phys. 22 (1951) 1350-1355. doi:10.1063/1.1699865.

[14] J. Wang, N. Li, O. Anderoglu, X. Zhang, A. Misra, J.Y. Huang, et al., Detwinning mechanisms for growth twins in face-centered cubic metals, Acta Materialia. 58 (2010) 2262-2270.

[15] D.L. Olmsted, S.M. Foiles, E.A. Holm, Survey of computed grain boundary properties in face-centered cubic metals: I. Grain boundary energy, Acta 
Materialia. 57 (2009) 3694-3703. doi:10.1016/j.actamat.2009.04.007.

[16] A.D. Banadaki, S. Patala, An efficient algorithm for computing the primitive bases of a general lattice plane, Journal of Applied Crystallography. 48 (2015) 585-588.

[17] E.R. Homer, Investigating the mechanisms of grain boundary migration during recrystallization using molecular dynamics, in: Risø Symposium, IOP Publishing, Roskilde, 2015: p. 012006. doi:10.1088/1757-899X/89/1/012006.

[18] S. Patala, C.A. Schuh, Symmetries in the representation of grain boundary-plane distributions, Philos Mag. 93 (2013) 524-573. doi:10.1080/14786435.2012.722700.

[19] E.R. Homer, S. Patala, J.L. Priedeman, Grain Boundary Plane Orientation Fundamental Zones and Structure-Property Relationships, Sci. Rep. 5 (2015) 15476. doi:10.1038/srep15476.

[20] S.M. Foiles, J.J. Hoyt, Computation of grain boundary stiffness and mobility from boundary fluctuations, Acta Materialia. 54 (2006) 3351-3357. doi:10.1016/j.actamat.2006.03.037.

[21] K.G. Janssens, D.L. Olmsted, E.A. Holm, S.M. Foiles, S.J. Plimpton, P.M. Derlet, Computing the mobility of grain boundaries, Nat Mater. 5 (2006) 124-127. doi:10.1038/nmat1559.

[22] J.A. Zimmerman, C.L. Kelchner, P.A. Klein, J.C. Hamilton, S.M. Foiles, Surface step effects on nanoindentation, Phys Rev Lett. 87 (2001) art. no.-165507.

[23] W. Read, W. Shockley, Dislocation Models of Crystal Grain Boundaries, Phys Rev. 78 (1950) 275-289.

[24] D. Wolf, A read-shockley model for high-angle grain boundaries, Scripta Metall Mater. 23 (1989) 1713-1718. doi:10.1016/0036-9748(89)90348-7.

[25] M.A. Tschopp, D.L. McDowell, Structures and energies of Sigma 3 asymmetric tilt grain boundaries in copper and aluminium, Philos Mag. 87 (2007) 3147-3173. doi:10.1080/14786430701255895.

[26] M.A. Tschopp, S.P. Coleman, D.L. McDowell, Symmetric and asymmetric tilt grain boundary structure and energy in $\mathrm{Cu}$ and $\mathrm{Al}$ (and transferability to other fcC metals), Integrating Materials and Manufacturing Innovation 2015 4:1. 4 (2015) 11. doi:10.1186/s40192-015-0040-1.

[27] C.J. O'Brien, S.M. Foiles, Hydrogen segregation to inclined twin grain boundaries in nickel, Philos Mag. 96 (2016) 2808-2828. doi:10.1080/14786435.2016.1217094.

[28] C.J. O'Brien, S.M. Foiles, Exploration of the mechanisms of temperaturedependent grain boundary mobility: search for the common origin of ultrafast grain boundary motion, J Mater Sci. 51 (2016) 6607-6623. doi:10.1007/s10853016-9944-1.

[29] D.L. Olmsted, L.G. Hector Jr, W.A. Curtin, R.J. Clifton, Atomistic simulations of dislocation mobility in Al, Ni and Al/Mg alloys, 13 (2005) 371-388. doi:10.1088/0965-0393/13/3/007.

[30] M.A. Meyers, L.E. Murr, A model for the formation of annealing twins in FCC metals and alloys, Acta Metall Mater. 26 (1978) 951-962.

[31] D.L. Medlin, C.B. Carter, J.E. Angelo, M.J. Mills, Climb and glide of a/3 〈111〉 dislocations in an aluminium $\Sigma=3$ boundary, Philosophical Magazine A. 75 (1997) 
733-747. doi:10.1080/01418619708207199.

[32] L. Liu, J. Wang, S.K. Gong, S.X. Mao, High Resolution Transmission Electron Microscope Observation of Zero-Strain Deformation Twinning Mechanisms in Ag, Phys Rev Lett. 106 (2011) 175504. doi:10.1103/PhysRevLett.106.175504.

[33] J. Wang, N. Li, A. Misra, Structure and stability of $\Sigma_{3}$ grain boundaries in face centered cubic metals, Philos Mag. 93 (2013) 315-327. doi:10.1080/14786435.2012.716908.

[34] J.H. Brunton, M.P.W. Wilson, The Kinetics of Twinning in Zinc and Tin Crystals, Proc. R. Soc. A. 309 (1969) 345-361. doi:10.1098/rspa.1969.0046.

[35] E. Faran, D. Shilo, Twin motion faster than the speed of sound, Phys Rev Lett. 104 (2010) 155501.

[36] J.G. Brons, H.A. Padilla II, G.B. Thompson, B.L. Boyce, Cryogenic indentationinduced grain growth in nanotwinned copper, Scripta Mater. 68 (2013) 781-784. doi:10.1016/j.scriptamat.2012.12.026.

[37] J.G. Brons, J.A. Hardwick, H.A. Padilla II, K. Hattar, G.B. Thompson, B.L. Boyce, The role of copper twin boundaries in cryogenic indentation-induced grain growth, Materials Science \& Engineering A. 592 (2014) 182-188. doi:10.1016/j.msea.2013.11.005.

[38] F. Ulomek, V. Mohles, Separating grain boundary migration mechanisms in molecular dynamics simulations, Acta Materialia. 103 (2016) 424-432. doi:10.1016/j.actamat.2015.10.021.

[39] J. Humberson, E.A. Holm, The structure and motion of incoherent $\Sigma_{3}$ grain boundaries in FCC metals, Acta Materialia. (n.d.) Under Review.

[40] A.D. Banadaki, S. Patala, A simple faceting model for the interfacial and cleavage energies of $\Sigma_{3}$ grain boundaries in the complete boundary plane orientation space, Comp Mat Sci. 112 (2016) 147-16o. doi:10.1016/j.commatsci.2015.09.062.

[41] D.L. Medlin, S.M. Foiles, C.B. Carter, Grain Boundary Dislocation Structure and Motion in an Aluminum $\Sigma=3$ [0-11] Bicrystal, MRS Proc. 466 (1996) 125. doi:10.1557/PROC-466-125.

[42] P.R. Cantwell, E.A. Holm, M.P. Harmer, M.J. Hoffmann, Anti-thermal behavior of materials, Scripta Mater. 103 (2015) 1-5. doi:10.1016/j.scriptamat.2015.02.011.

[43] V. Sursaeva, B. Straumal, Influence of grain boundary inclination on the grain boundary and triple junction motion in Zn, Materialwissenschaft Und Werkstofftechnik. 36 (2005) 528-532. doi:10.1002/mawe.200500929.

[44] M.Y. Gutkin, K.N. Mikaelyan, I.A. Ovid'ko, Athermal grain growth through cooperative migration of grain boundaries in deformed nanomaterials, Scripta Mater. 58 (2008) 850-853. doi:10.1016/j.scriptamat.2008.01.002.

[45] M.I. Mendelev, C. Deng, C.A. Schuh, D.J. Srolovitz, Comparison of molecular dynamics simulation methods for the study of grain boundary migration, Model Simul Mater Sc. 21 (2013) 045017. doi:10.1088/0965-0393/21/4/045017.

[46] S.P. Coleman, D.E. Spearot, S.M. Foiles, Computational Materials Science, Comp Mat Sci. 86 (2014) 38-42. doi:10.1016/j.commatsci.2014.01.022.

[47] Z.T. Trautt, M. Upmanyu, A. Karma, Interface mobility from interface random walk, Science. 314 (2006) 632-635. doi:10.1126/science.1131988. 
[48] C. Deng, C.A. Schuh, Atomistic Simulation of Slow Grain Boundary Motion, Phys Rev Lett. 106 (2011) -. doi:10.1103/PhysRevLett.106.045503.

[49] F. Ulomek, C.J. O'Brien, S.M. Foiles, V. Mohles, Energy conserving orientational force for determining grain boundary mobility, Model Simul Mater Sc. 23 (2015) 025007.

[50] J. Humberson, E.A. Holm, Anti-thermal mobility in the $\Sigma_{3}[111] 60^{\circ}\{1185\}$ grain boundary in nickel: Mechanism and computational considerations, Scripta Mat. 130 (2017) 1-6. doi:10.1016/j.scriptamat.2016.10.032.

[51] V.A. Al'shitz, V.L. Indenbom, Dynamic dragging of dislocations, Sov Phys-Usp. 18 (1975) 1-20.

[52] A.D. Brailsford, Phonon Component of Dislocation Drag, J Appl Phys. 41 (1970) 4439. doi:10.1063/1.1658478.

[53] A.D. Brailsford, Electronic component of dislocation drag in metals, Phys Rev. 186 (1969) 959.

[54] V.I. Alshits, The phonon-dislocation interaction and its role in dislocation dragging and thermal resistivity, in: V.L. Indenbom, J. Lothe (Eds.), Elastic Strain Fields and Dislocation Mobility, North-Holland, Amsterdam, 1992: pp. 625-698.

[55] A.Y. Kuksin, A.V. Yanilkin, Atomistic simulation of the motion of dislocations in metals under phonon drag conditions, Phys Solid State. 55 (2013) 1010-1019. doi:10.1134/S1063783413050193.

[56] C. Kittel, Introduction to Solid State Physics, John Wiley \& Sons, Hoboken, 2005.

[57] C. Lane, Wave Propagation in Anisotropic Media, in: The Development of a 2D Ultrasonic Array Inspection for Single Crystal Turbine Blades, Springer International Publishing, Switzerland, 2014: pp. 13-39. doi:10.1007/978-3-31902517-9_2.

[58] H. Zhang, M. Upmanyu, D.J. Srolovitz, Curvature driven grain boundary migration in aluminum: molecular dynamics simulations, Acta Materialia. 53 (2005) 79-86. doi:10.1016/j.actamat.2004.09.004.

[59] C.P. Race, Quantifying uncertainty in molecular dynamics simulations of grain boundary migration, Mol Simulat. 41 (2014) 1069-1073. doi:10.1080/08927022.2014.935774.

[6o] C.P. Race, J. von Pezold, J. Neugebauer, Role of the mesoscale in migration kinetics of flat grain boundaries, Phys Rev B. 89 (2014). doi:10.1103/PhysRevB.89.214110.

[61] R. Hadian, B. Grabowski, C.P. Race, J. Neugebauer, Atomistic migration mechanisms of atomically flat, stepped, and kinked grain boundaries, Phys Rev B. 94 (2016) 165413-10. doi:10.1103/PhysRevB.94.165413. 


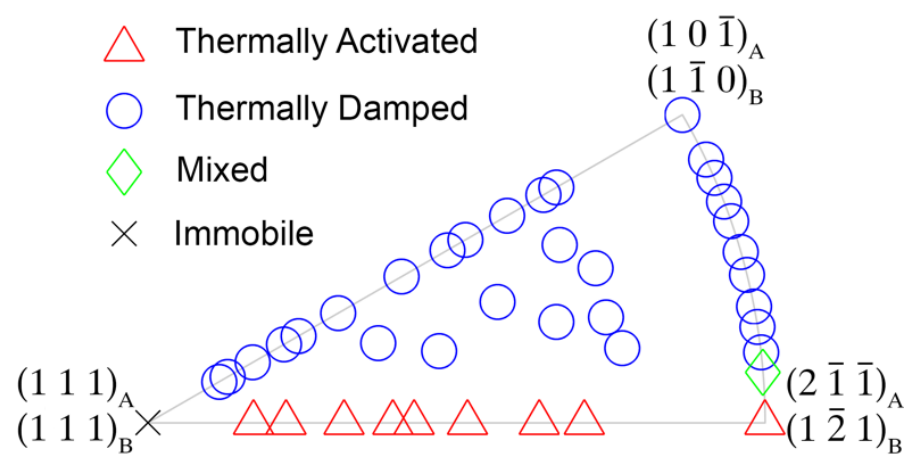

Figure 1 Temperature-dependent GB mobility trends plotted in the GB plane orientation fundamental zone. The vertices of the fundamental zone are defined for both crystals, A and B, on either sides of the boundary plane. 
(a) Boundary Plane Indices From Both Crystals
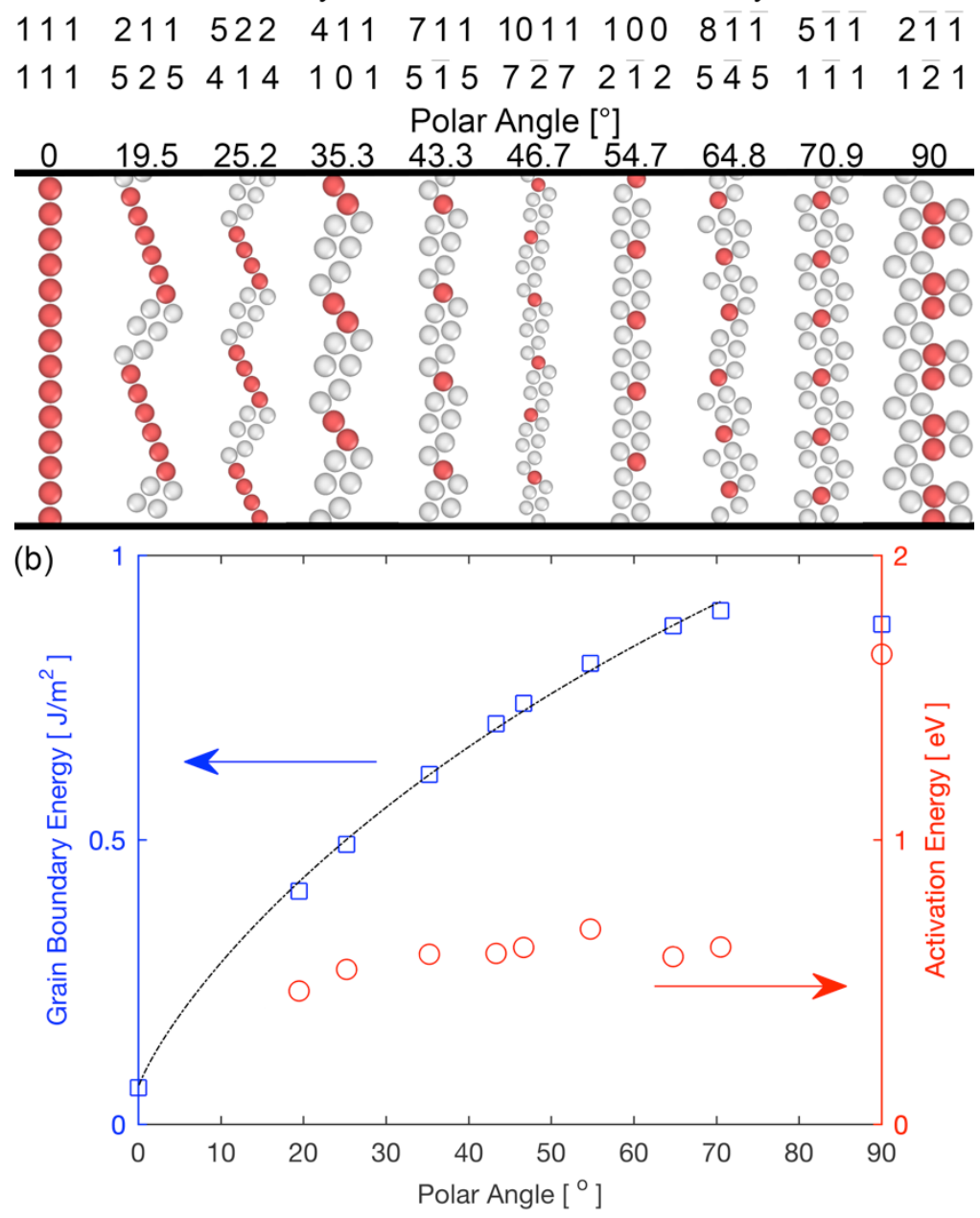

Figure 2(a) Atomic structure of thermally activated GBs identified by their boundary plane indices on both sides of the boundary plane (side A normals on the top row, B on the bottom) and their polar angle relative to the coherent twin. (b) Plot of GB energy and activation energy as a function of their polar angle. The GB Energy is fitted with a Read-Shockley line that matches the data for all but the $90^{\circ}$ polar angle GB. 
(a)

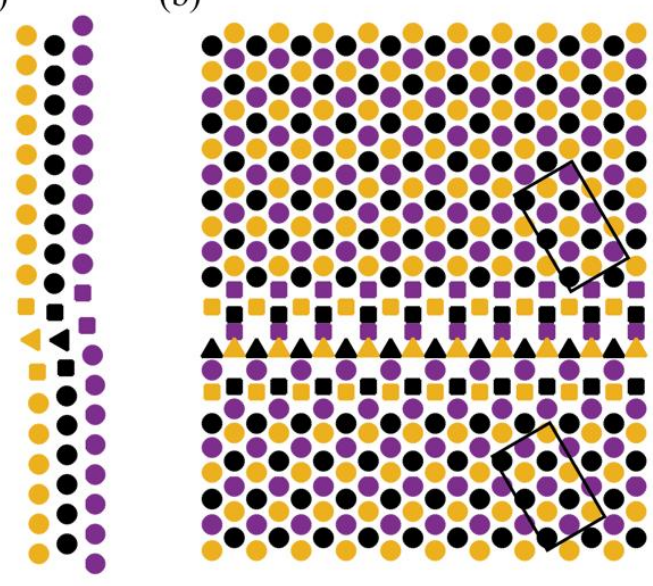

(c)

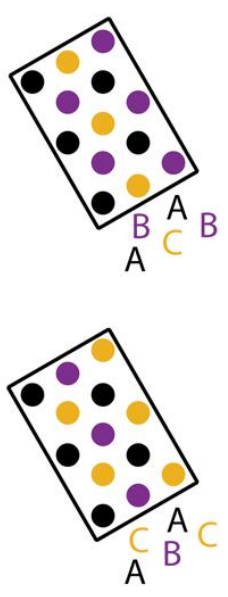

(d)

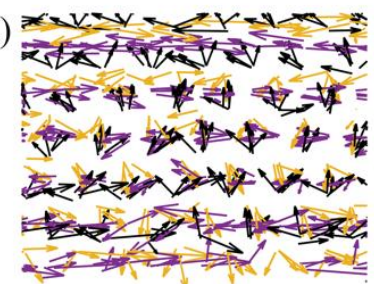

(e)

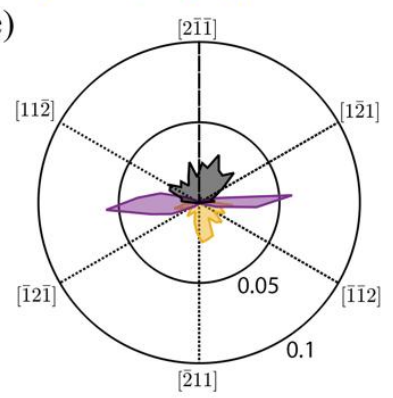

Figure 3(a) Atomic structure of the $(2 \overline{1} \overline{1}) \mathrm{GB}$ viewed down the [0 $\overline{1} 1]$ axis. Atoms are colored by atomic plane (A, B and C correspond to black, purple and yellow respectively). Atom shapes correspond to their categorization via common neighbor analysis: circles, triangles and squares represent atoms that have FCC, HCP, and no defined crystal structure, respectively. (b) Atomic structure of the (2 $\overline{1} \overline{1}$ ) GB viewed down the [1 111 ] axis. (c) Magnified views of the top and bottom grains to show ordering difference of the $\mathrm{ABC}$ planes across the $\mathrm{GB}$; note the reversal of the $\mathrm{C}$ and $\mathrm{B}$ planes between the two grains. (d) Plot of slip vector directions for the migration of the $\left(\begin{array}{lll}2 & \overline{1} & \overline{1}\end{array}\right) \mathrm{GB}$ viewed down the $\left[\begin{array}{lll}1 & 1 & 1\end{array}\right]$ axis. (e) Distribution of angles of the slip vector analysis separated by atomic plane stacking $(A, B, C)$. The concentric circles indicate the fraction of all slipping atoms with vectors in the specified direction. The dashed line indicates the direction of GB migration and the dotted lines indicate the directions of a Shockley partial $\left(\begin{array}{lll}1 & 1 & 2\end{array}\right\rangle$ directions in the (1 111$)$ plane). For panels (b) through (e) directions are as given in (e) with [ $\left[\begin{array}{lll}2 & \overline{1}\end{array}\right]$ vertical and [ $\left[\begin{array}{ll}0 & 1\end{array}\right]$ horizontal.

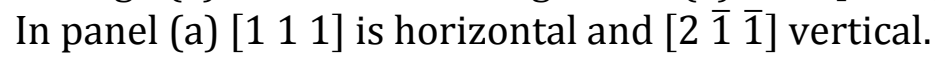




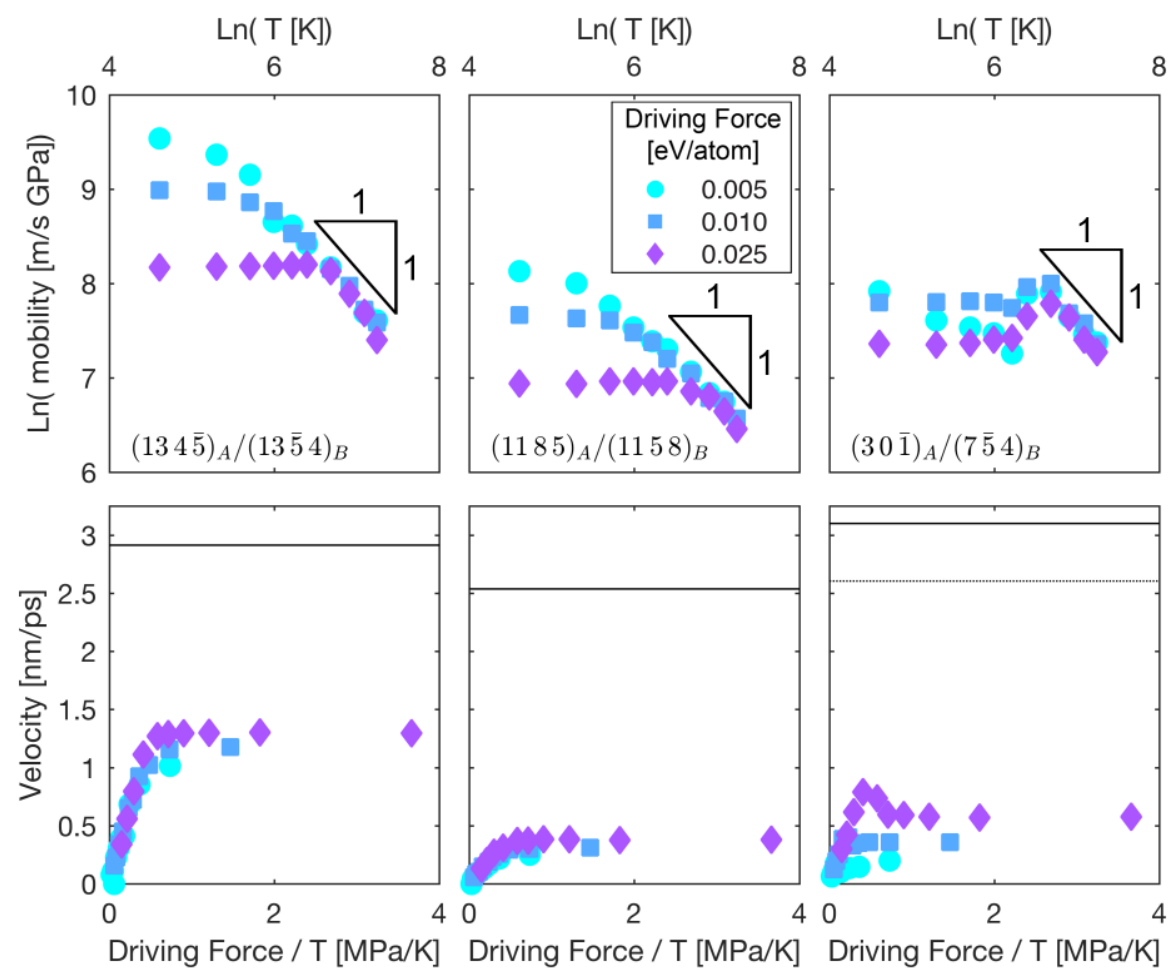

Figure 4 Temperature-dependent mobility plots and velocity vs driving force/temperature plots for 3 different $\Sigma 3$ boundary plane orientations. Each GB has inverse temperature dependence of the mobility at high temperatures and athermal mobility at low temperatures. The influence of driving force is indicated by the different colors and symbols, as denoted by the key. It is important to note that low driving forces lead to higher mobility at low temperatures, though this still corresponds to a lower velocity, as indicated in the lower row of plots. The solid line and dashed lines in the lower row of plots correspond to the lowest wave speed for those GBs. 
(a)

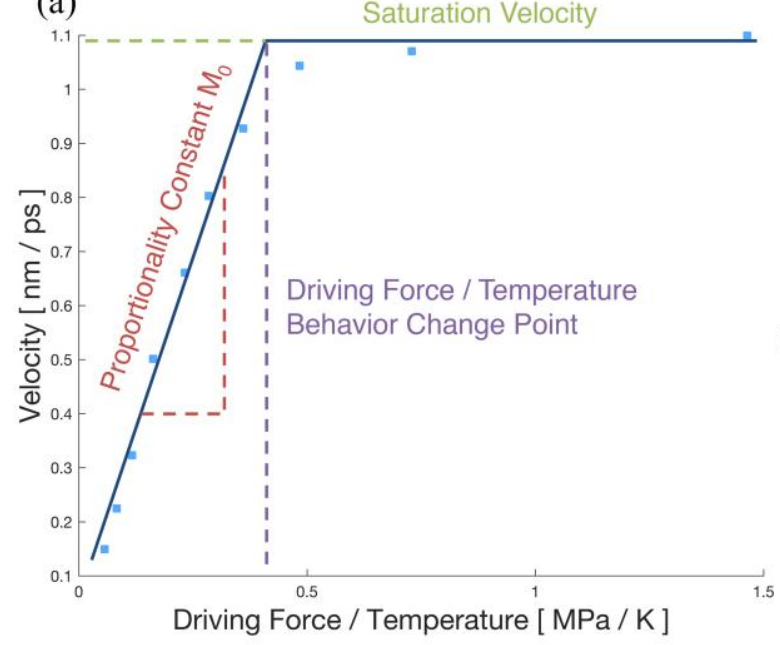

(c)

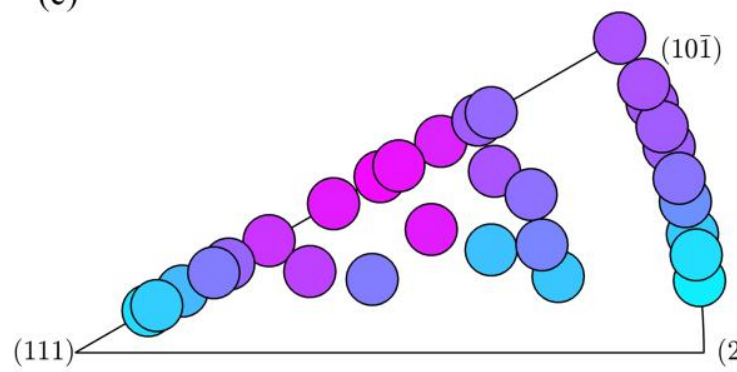

$(2 \overline{1} \overline{1})(111)$ (b)

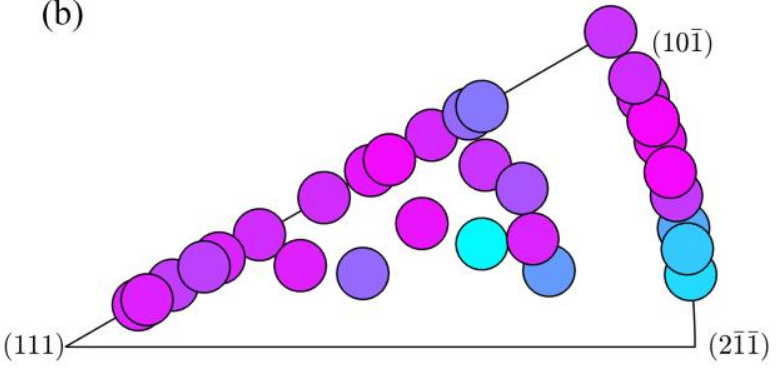

(d)
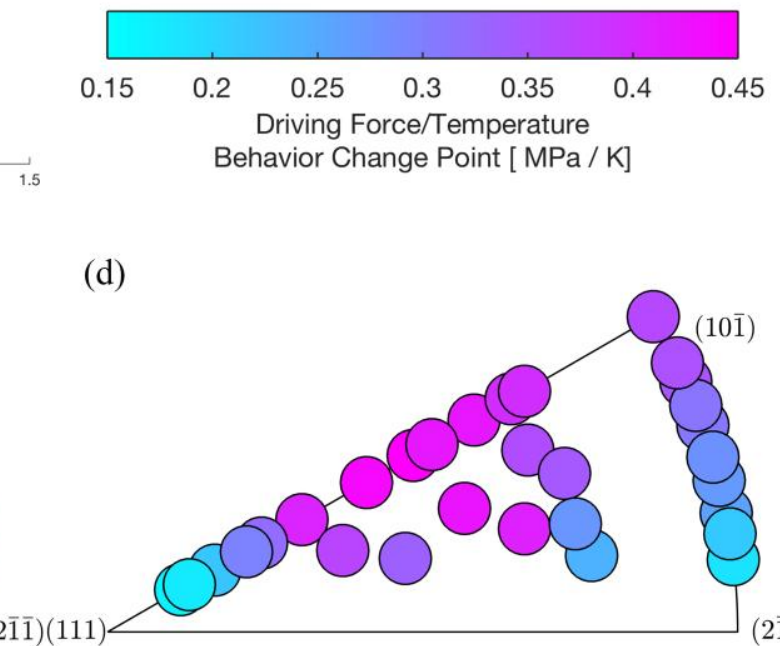

$(2 \overline{1} \overline{1})$
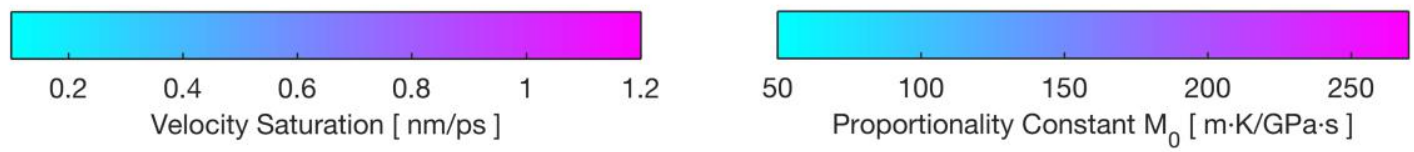

Figure 5 (a) Illustrative example depicting features of the velocity vs. driving force plot, that can be analyzed and compared for all the thermally damped GBs. These three features are plotted for the thermally damped GBs in the fundamental zone in (b-d). (b) Plot of the driving force/temperature behavior change point where the GB changes from thermally damped to athermal mobility. (c) Plot of the magnitude of the velocity saturation at high values of driving force/temperature. (d) Plot of the proportionality constant, $\mathrm{M}_{0}$ for the same GBs. Note the crystallographic dependence of (c) and (d) and the lack thereof for (b). 
(a)

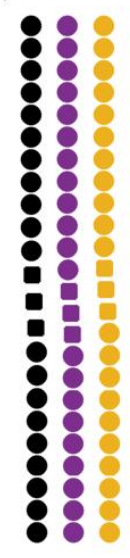

(b)

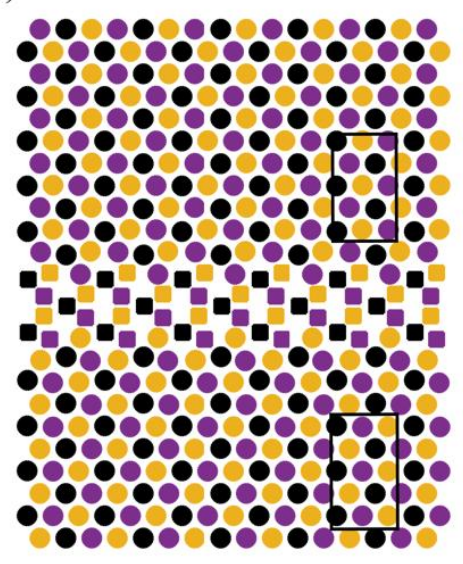

(c)
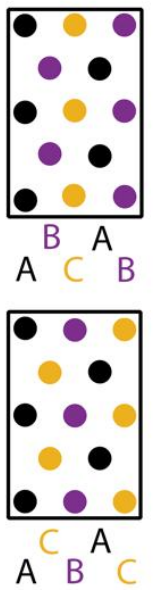

(d)

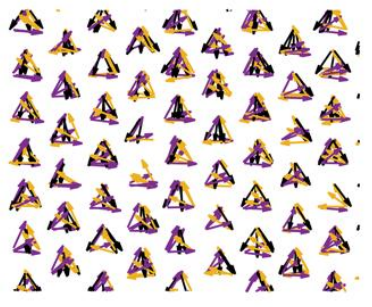

(e)

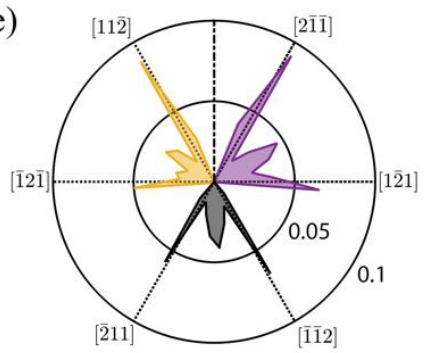

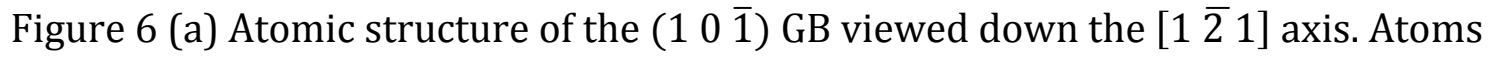
are colored by atomic plane (A, B and C correspond to black, purple and yellow respectively) Atom shapes correspond to their categorization via common neighbor analysis: circles, triangles and squares represent atoms that have FCC, HCP, and no defined crystal structure, respectively. (b) Atomic structure of the (10 $10 \overline{1}$ ) GB viewed down the [1 111 ] axis. (c) Magnified views of the top and bottom grains to show ordering difference of the $\mathrm{ABC}$ planes across the $\mathrm{GB}$; note the reversal of the $\mathrm{C}$ and $\mathrm{B}$ planes between the two grains. (d) Plot of slip vector directions for the migration of the $\left(\begin{array}{lll}1 & 0 & \overline{1}\end{array}\right)$ GB viewed down the [ $\left[\begin{array}{lll}1 & 1 & 1\end{array}\right]$ axis. (e) Distribution of angles of the slip vector analysis separated out by atomic planes. The concentric circles indicate the fraction of all slipping atoms with vectors in the specified direction. The dashed line indicates the direction of GB migration and the dotted lines indicate the directions of a Shockley partial $\left(\begin{array}{llll}1 & 1 & 2\end{array}\right\rangle$ directions in the ( $\left.\begin{array}{lll}1 & 1 & 1\end{array}\right)$ plane). For panels (b) through (e)

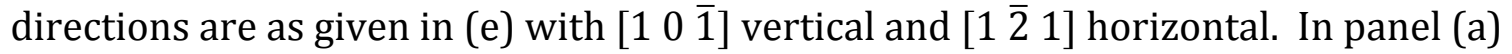
$\left[\begin{array}{lll}1 & 1 & 1\end{array}\right]$ is horizontal and [ $\left[\begin{array}{lll}1 & 0 & \overline{1}\end{array}\right]$ vertical. 

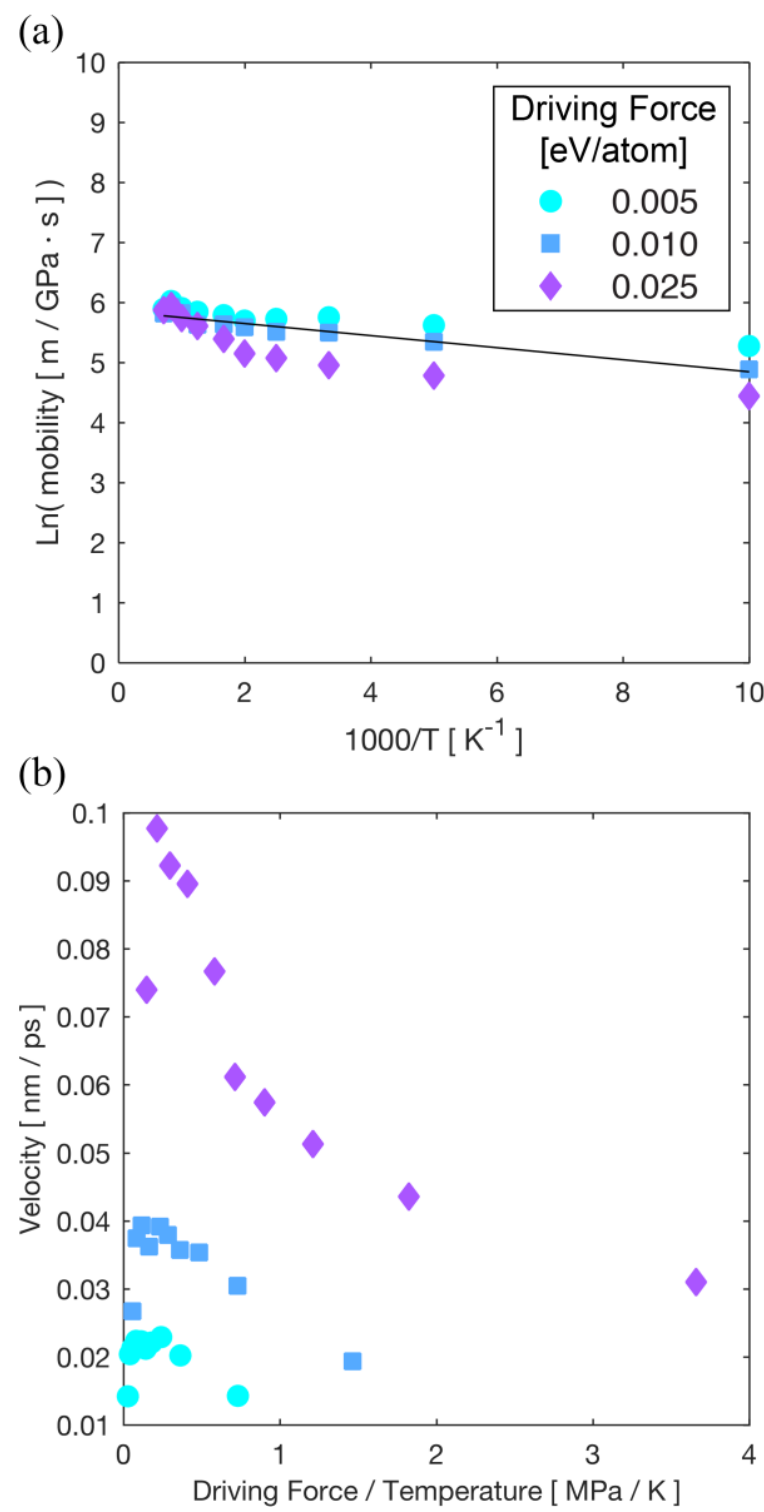

Figure 7 (a) Arrhenius plot of GB mobility for the (7 $\overline{3} \overline{4})$ GB. The Arrhenius fit is plotted as a solid line and has an activation energy of $0.009 \mathrm{eV}$. The mobility also has characteristics of thermally damped migration in that lower driving forces lead to higher mobility values. The influence of driving force is indicated by the different colors and symbols, as denoted by the key. (b) Plot of GB velocity as a function of driving force/temperature. Note the large spike in velocity at low values of driving force/temperature. 
(a)

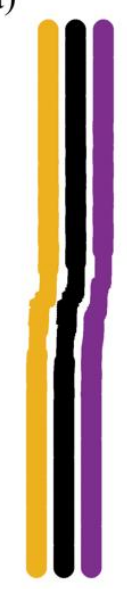

(b)

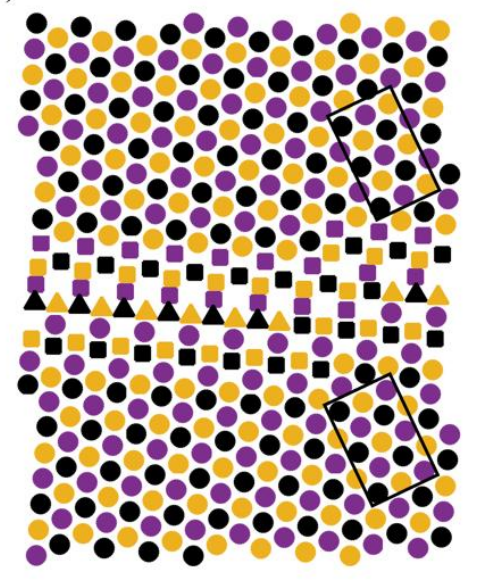

(c)

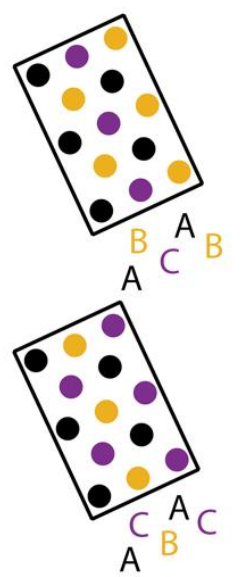

(d)

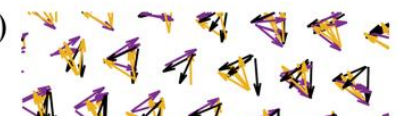

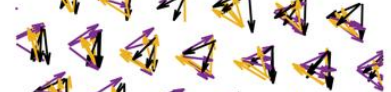

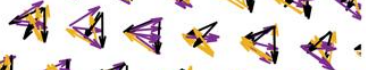
त $4<14$ $4+4+2<$

(e)

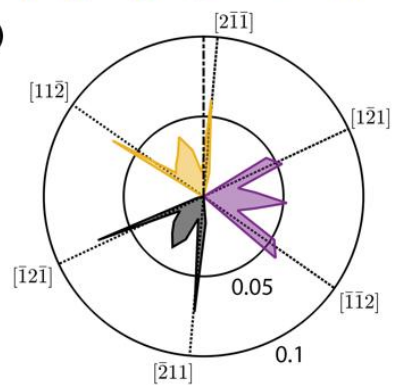

Figure 8 (a) Atomic structure of the $(7 \overline{3} \overline{4})$ GB viewed down the [1 $\overline{11} 10]$ axis. Atoms are colored by atomic plane (A, B and C correspond to black, purple and yellow respectively) Atom shapes correspond to their categorization via common neighbor analysis: circles, triangles and squares represent atoms that have FCC, HCP, and no defined crystal structure, respectively. (b) Atomic structure of the ( $7 \overline{3} \overline{4}$ ) GB viewed down the [1 111 ] axis. (c) Magnified views of the top and bottom grains to show ordering difference of the $\mathrm{ABC}$ planes across the GB; note the reversal of the $\mathrm{C}$ and $\mathrm{B}$ planes between the two grains. (d) Plot of slip vector directions for the migration of

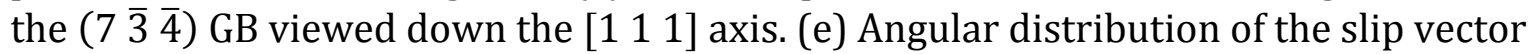
analysis separated out by atomic planes. The concentric circles indicate the fraction of all slipping atoms with vectors in the specified direction. The dashed line indicates the direction of GB migration and the dotted lines indicate the directions of a Shockley partial $\left(\left\langle\begin{array}{lll}1 & 1 & 2\rangle\end{array}\right)\right.$ directions in the (1 111$)$ plane). For panels (b) through (e) directions

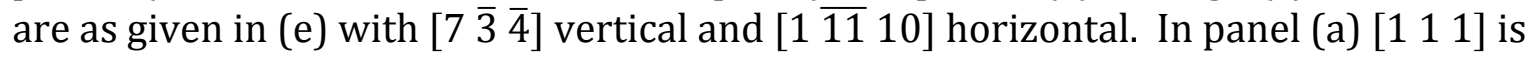

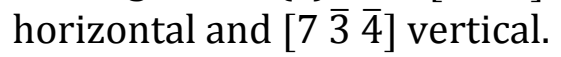




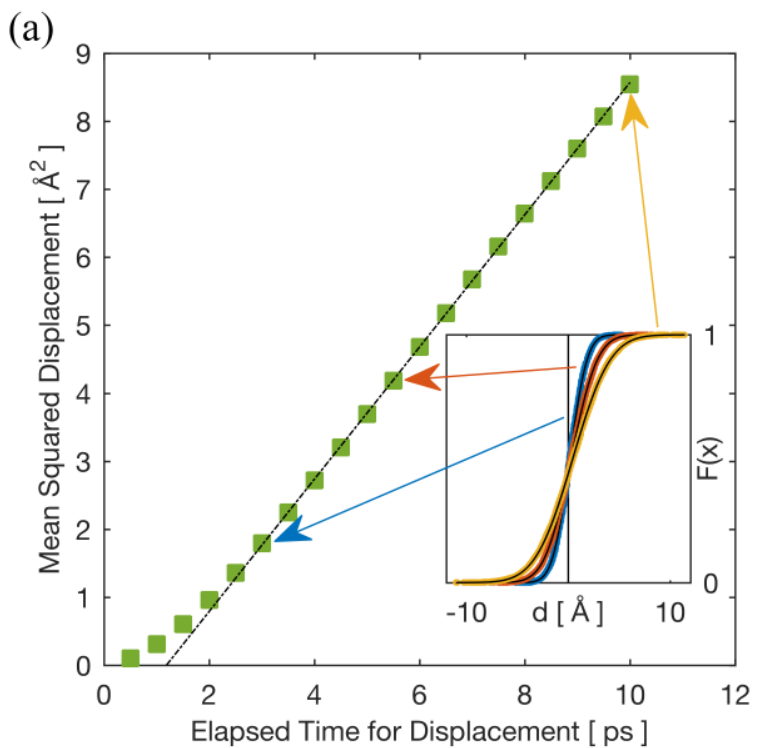

(b)

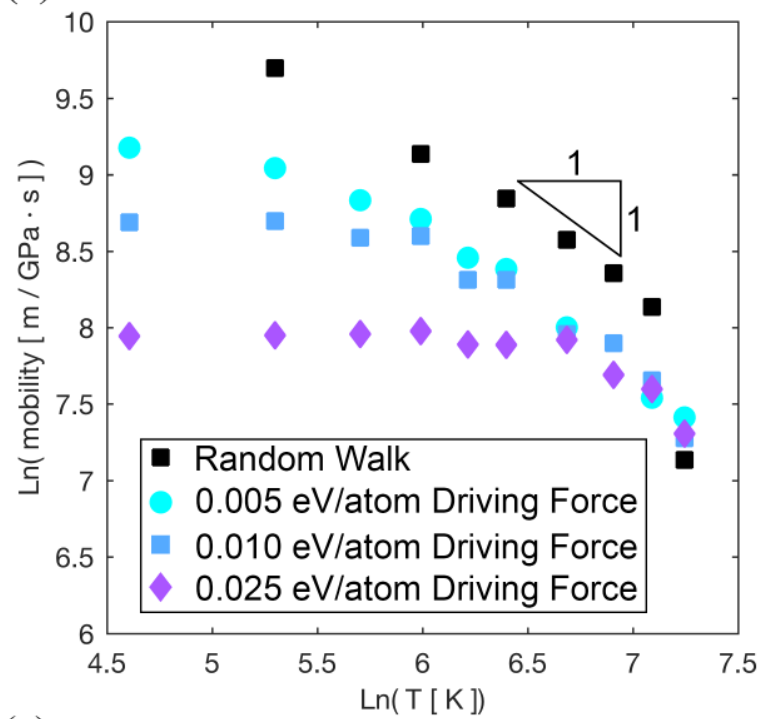

(c)

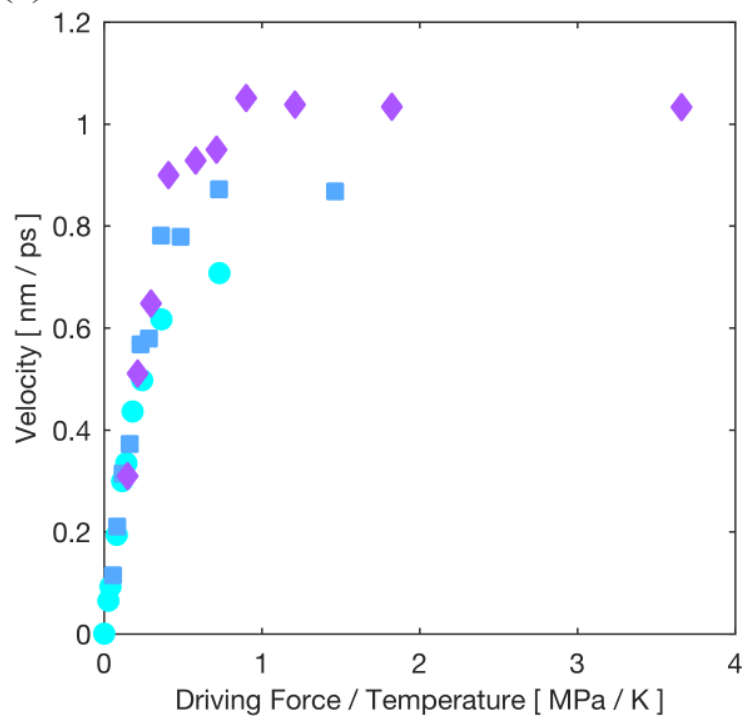


Figure 9 (a) Mean squared mean displacement of a GB, $\left\langle\bar{x}^{2}\right\rangle$, as a function of time for a random walk analysis of the $\left(\begin{array}{lll}1 & 0 & \overline{1}\end{array}\right) \mathrm{GB}$ at $200 \mathrm{~K}$. Cumulative distribution plots, overlaid with a cumulative distribution function line, are shown in an inset for three of the $\left\langle\bar{x}^{2}\right\rangle$. Together, the plot of $\left\langle\bar{x}^{2}\right\rangle$ and the distributions indicate a good measure of the mobility by random walk analysis. (b) Plot comparing mobility obtained by the synthetic driving force method with mobility obtained from the random walk method for the $\left(\begin{array}{lll}1 & 0 & \overline{1}\end{array}\right) \mathrm{GB}$. The influence of driving force is indicated by the different colors and symbols, as denoted by the key. (c) Plot of GB velocities as a function of driving force/temperatures for the (1 0 1) GB; random walk analysis has zero driving force so it cannot be plotted here, though it is in the zero driving force limit, which would correspond to the linear portion at low driving force/temperature. 


\section{Crystallographically Dependent $\Sigma 3$ Grain Boundary Migration Mechanisms}

\section{Crystallography}

$\triangle$ Thermally Activated

Thermally Damped

Mixed

$\times$ Immobile

$\left(\begin{array}{lll}1 & 1 & 1\end{array}\right)$

$\left(\begin{array}{lll}1 & 1 & 1\end{array}\right)_{\mathrm{B}}^{\mathrm{A}} \mathrm{X}$

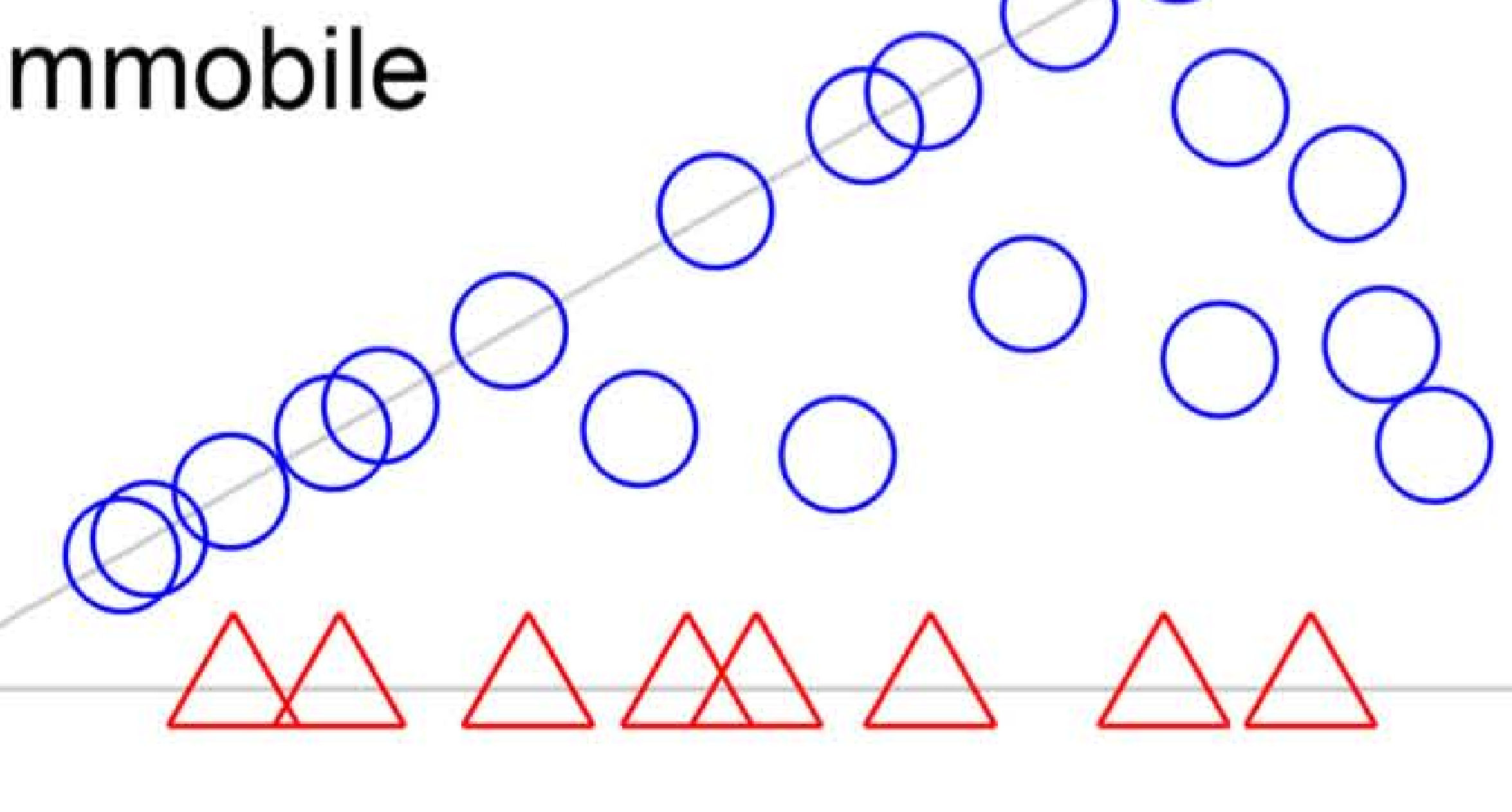

\section{Temperature Dependence}

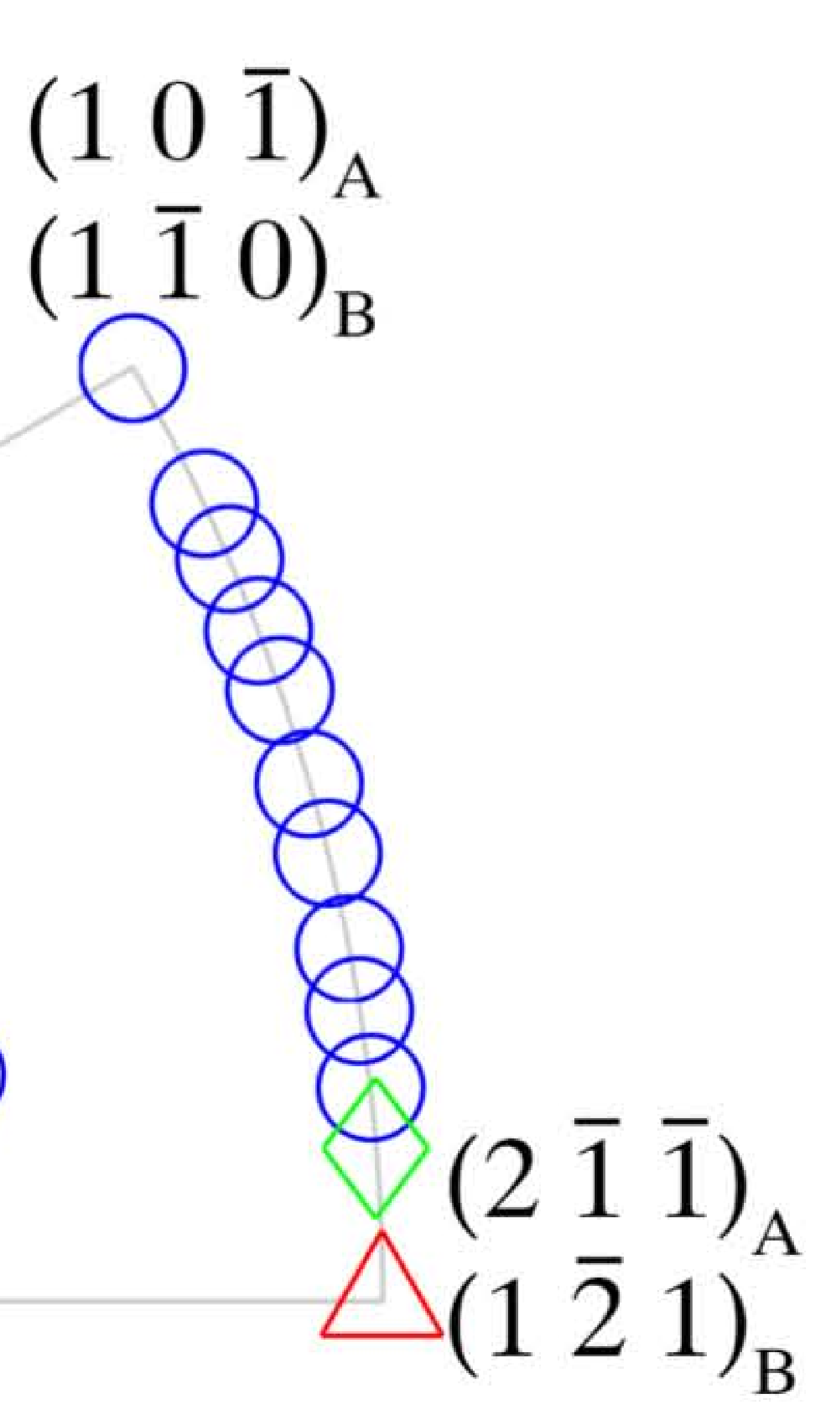

Mechanisms

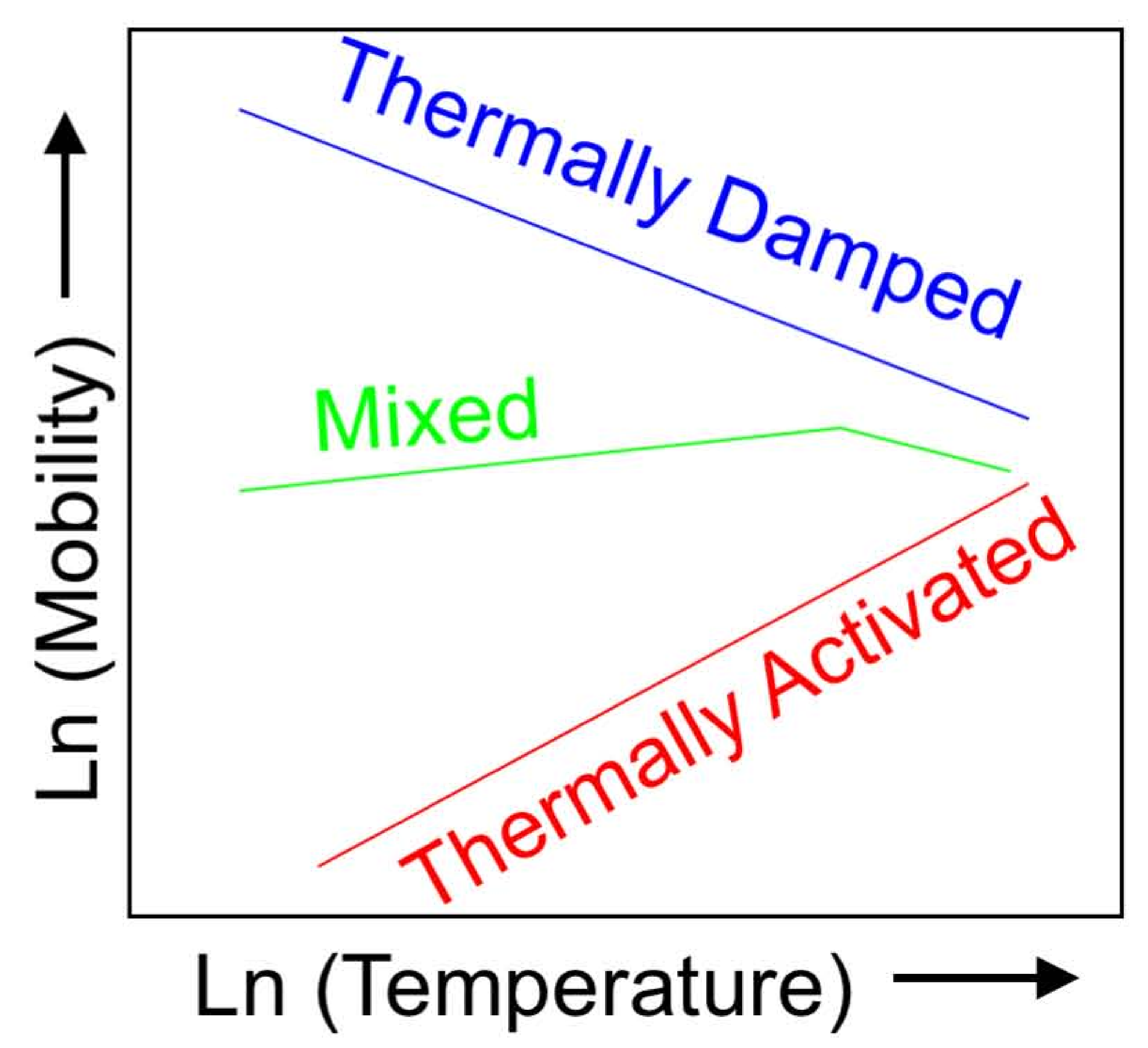

Thermally Damped \& Mixed Ordered Migration

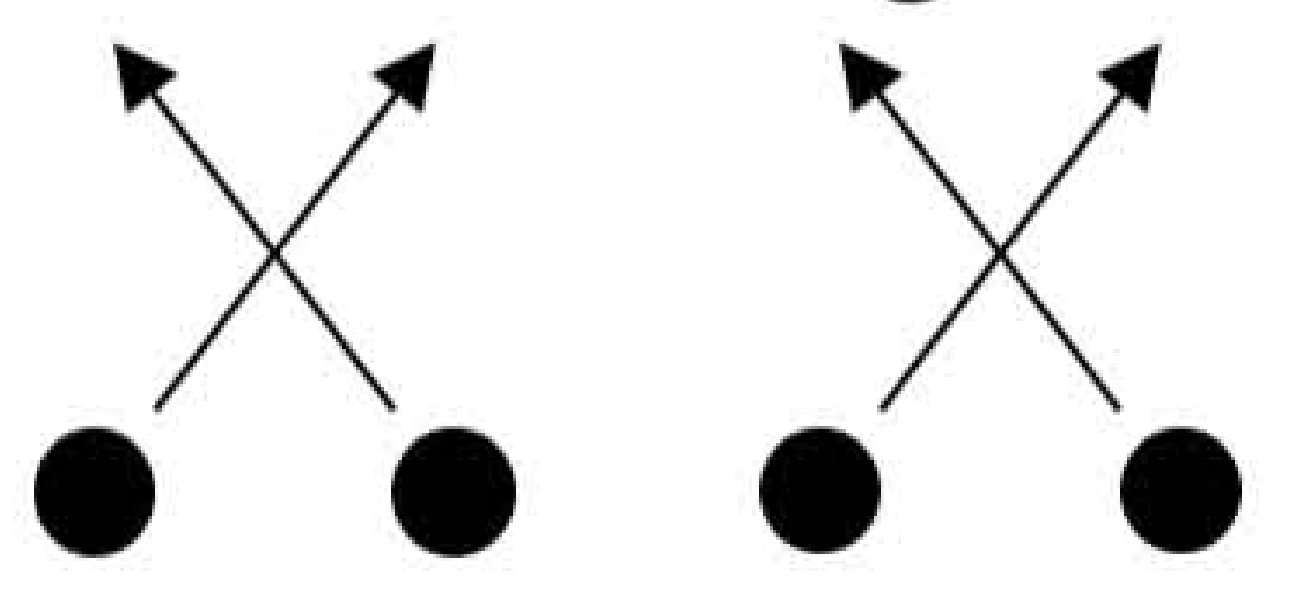

Thermally Activated Random Migration

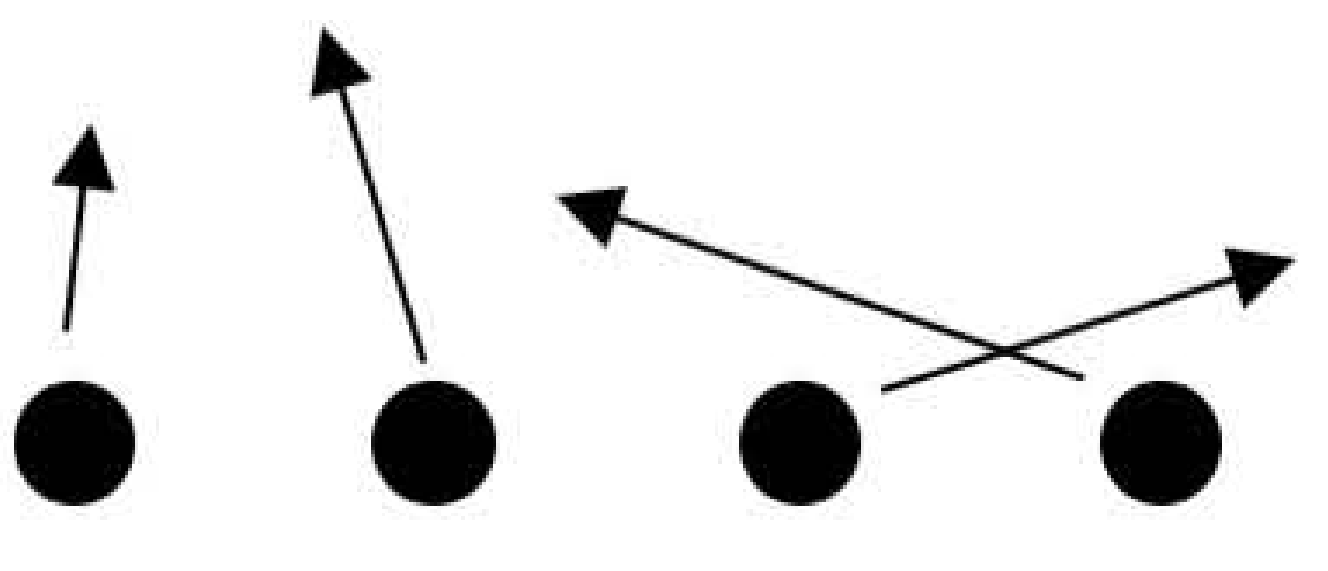

